\title{
Distributed Cooperative Control for Economic Operation of Multiple Plug-in Electric Vehicle Parking Decks
}

Shengyao Xu, Hajir Pourbabak, and Wencong Su (Corresponding Author)

Department of Electrical and Computer Engineering

University of Michigan-Dearborn

4901 Evergreen Road, Dearborn, Michigan 48128, U.S.A.

Emails: shengyao@umich.edu; hpourbab@umich.edu; wencong@umich.edu

Phone: $+1-313-593-5314 \quad$ Fax: $+1-313-583-6336$

\section{Summary}

This paper addresses the economic operation of multiple plug-in electric vehicle (PEV) parking decks using novel distributed cooperative control methods. In this paper, we proposes a consensus-based distributed cooperative control algorithm to determine the best operation strategy for the operator/owner of multiple parking decks. The proposed distributed control approach completely eliminates the reliance on a central controller to make an optimal decision while still satisfying a variety of global and local constraints. Case studies demonstrate the accuracy, robustness, effectiveness, and scalability of the proposed distributed cooperative control method for solving the economic operation problem of multiple PEV parking decks under various operating conditions. The distributed control ideas contained here can apply to many other smart grid applications.

Keywords: Economic Operation, Distributed Consensus Algorithm, Cooperative Control, Plug-in Electric Vehicle (PEV), Smart Grid

This is the author manuscript accepted for publication and has undergone full peer review but has not been through the copyediting, typesetting, pagination and proofreading process, which may lead to differences between this version and the Version of Record. Please cite this article as doi: 10.1002 /etep. 2348

This article is protected by copyright. All rights reserved. 


\section{Introduction}

In recent years, electrified transportation systems have been drawing increasing attention with the growing urgency to reduce carbon emission, improve energy efficiency, and promote sustainability. Advances in plug-in electric vehicle (PEV) technologies offer great promise to revolutionize future electrified transportation systems. Around the world, a tremendous amount of effort has been made to boost the market penetration of PEVs because of their carbon emissions, energy independence, and high fuel economy [1], [2], [3]. Ideally, PEVs can be recharged anytime and anywhere. Therefore, the successful deployment of PEVs is highly dependent upon the quality, availability, and affordability of the service that the critical physical infrastructure can provide [4]. The advanced control strategies will play a more critical role in supporting the deployment of PEV charging infrastructure [5].

The existing PEV charging control approaches can be divided into three categories, namely, centralized control, decentralized control, and distributed control. Table 1 summarizes the comparisons between the three categories.

Table 1: Comparison between Centralized, Decentralized, and Distributed Control

\begin{tabular}{|c|c|c|}
\hline 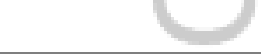 & Pros & Cons \\
\hline $\begin{array}{c}\text { Centralized } \\
\text { control }[6]-[11]\end{array}$ & $\begin{array}{l}\text { - Easy to implement } \\
\text { - Relatively low capital } \\
\text { cost }\end{array}$ & $\begin{array}{l}\text { - } \text { Computational burden } \\
\text { - Not easy to expand } \\
\text { - Single point of failure } \\
\text { - Requires high-bandwidth dedicated communication links }\end{array}$ \\
\hline $\begin{array}{c}\text { Decentralized } \\
\text { control [13]-[18] }\end{array}$ & $\begin{array}{l}\text { - Local information only } \\
\text { - No need for two-way } \\
\text { high-speed } \\
\text { communication }\end{array}$ & - Less chance to achieve global optimal solution \\
\hline & - Easy to expand & - Needs synchronization \\
\hline
\end{tabular}




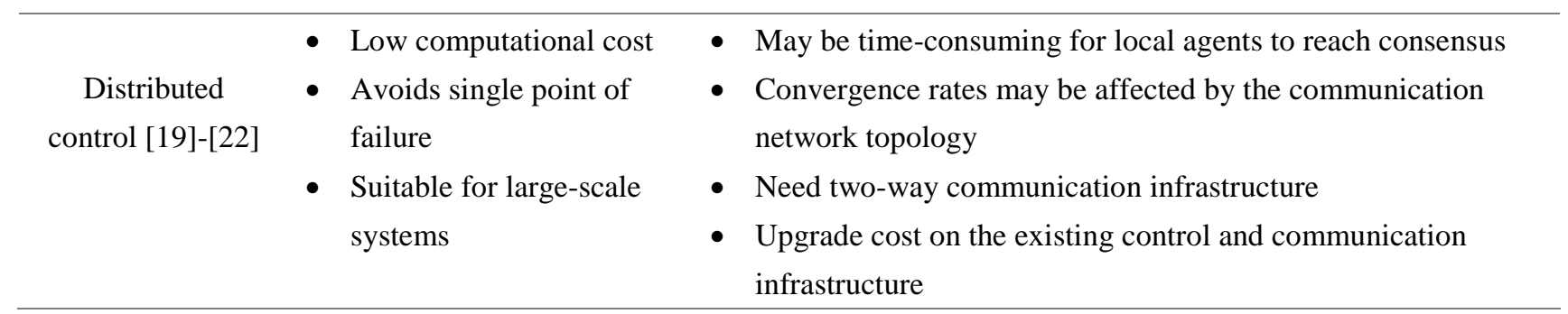

The majority of existing literature focuses on centralized control for PEV charging infrastructure. In most cases, the dedicated communication links are required to exchange data between the central controller (e.g., aggregator) and the local agents (e.g., PEV charging stations). For example, Luo et al. [6] proposed a centralized charging strategy of EVs for the high penetration of battery swapping stations in power systems. Xu et al. [7] proposed a centralized control framework and methodology for coordinated PEV charging considering a variety of charging requirements and local constraints. Jin et al. [8] investigated the impacts of large-scale EV charging taking into account the energy storage for aggregate energy transaction in a regulated electricity markets. Sundstrom et al. [9] proposed an optimal planning problem of centralized PEV charging scheme subject to various power grid constraints such as voltage. Khodayar et al. [10] proposed a stochastic security-constrained unit commitment (SCUC) model for the coordinated grid integration of PEV fleets and wind generation in power systems. Sattarpour et al. [11] proposed an optimal placement of parking lots with optimal scheduling in power systems to maximize the benefit considering different peak load states and different electricity prices. Badri et al. [12] proposed an optimal electricity framework for the operation of electricity provider considering EV charging load and time-of-use rates. 
Centralized control approaches are suitable for relatively small-scale systems without having to reconstruct the existing communication and control networks. However, as the market penetration of PEVs is continuously growing, there are some technical barriers to the centralized control of PEV charging, such as heavy computation burden and single point of failure.

Decentralized control is an intermediate solution to addressing the abovementioned challenges. The ultimate objective is to maximize the benefits of local agents without coordinating with others. Su et al. [13] proposed an Estimation of Distribution Algorithm-based algorithms to locally manage the power allocation at a municipal PEV parking deck. Guo et al. [14] proposed a day-ahead and real-time operation framework for a single PEV parking lot to determine the best retail electricity price and parking fee. Luo et al. proposed a decentralized charging control method to utilize the PEV charging loads to mitigate the wind power fluctuations in a decentralized manner [15]. He et al. [16] addressed an optimal scheduling problem to reduce the total operation cost of PEVs based on the local information only. Cao et al. [17] proposed a smart control method to manage EV charging loads taking into consideration the time-of-use price in a regulated market. D'hulst et al. [18] proposed a decentralized method to coordinate the charging of electric vehicles to avoid network congestion issues.

Fig. 1 illustrates the proposed economic operation of multiple PEV charging stations in a distributed control manner. 


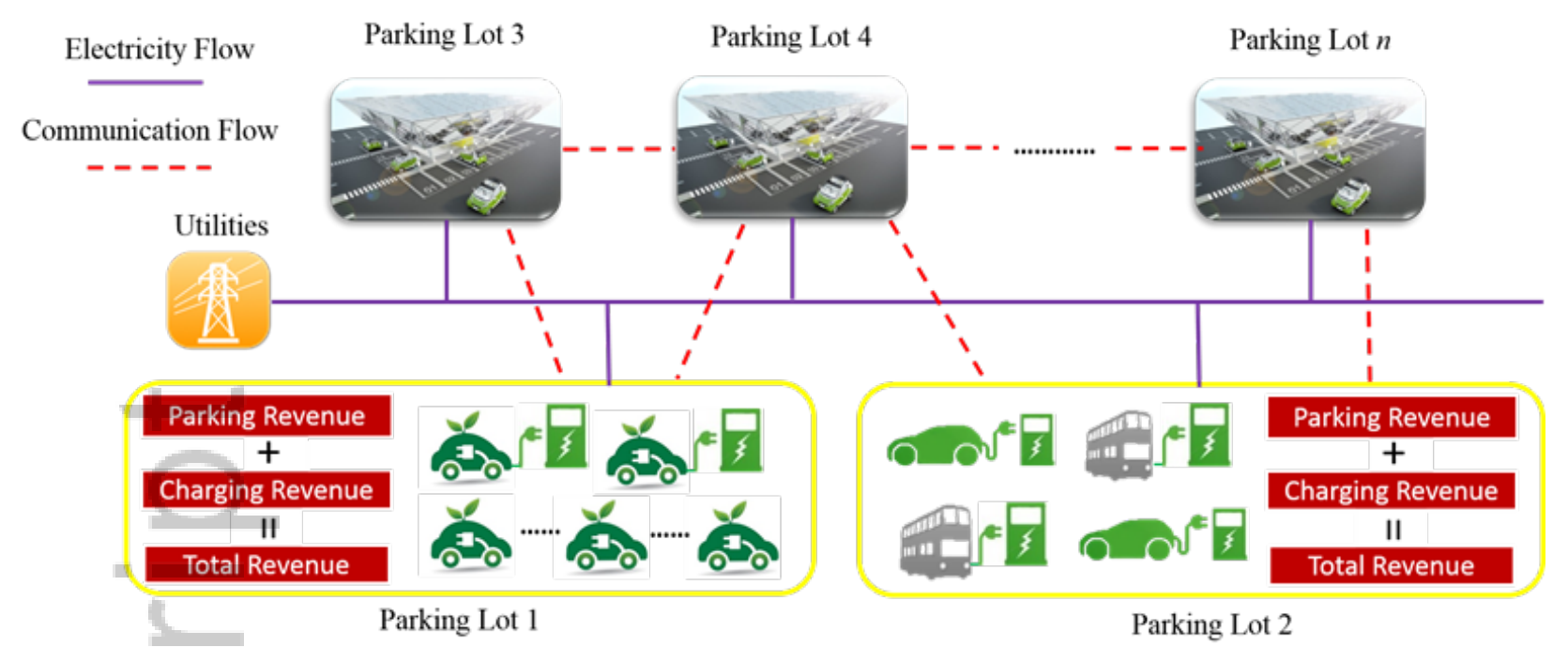

Fig. 1. An Illustrated Structure of Economic Operation of Multiple PEV Charging Stations in a Distributed Control Manner

Since there is no communication link between the different local agents, there is no guarantee that the decisions made by each local agent can contribute to the global optimal decision of the entire system. It is well known that the participating agents (e.g., PEV parking lots) may have partial or complete conflicts of interests, such as attracting more PEV customers to their own parking decks. Strong interactions between different agents may prevent the entire system from achieving global optimal operation. In general, the overall closed-loop performance of decentralized control is limited because of the lack of communication links and information exchange.

This leads to the emerging interest in distributed control, in which local agents can share information through two-way communication links in order to find the global optimal decision. For example, Wen et al. [19] developed a distributed control algorithm to determine the best PEV charging scenarios. $\mathrm{Xu}$ [20] used multi-agent concepts and consensus algorithms to optimally allocate the PEV charging power, as originally proposed in [13]. Rahbari-Asr et al. [21] developed 
a cooperative distributed algorithm for PEV charging control based on KKT conditions and consensus network theorem. Miranda et al. [22] proposed a multi-agent based implementation of a management system to automate the negotiation of electricity allocation for EV charging.

Distributed control has the potential to solve large-scale PEV charging problems. The majority of current research work focuses on the optimal management of PEV charging loads from either electric utility or customer perspectives, such as [18]-[22]. Compared to the existing literature work, our objective is to maximize the total revenue of the parking deck owner considering the charging fee and the parking rebate rate. It offers a dependable reference for the parking deck owners. To the best of our knowledge, the similar ideas have not been fully explored yet. Also, most research work is focused on centralized and decentralized methods to solve the optimal charging control problems [6]-[14]. In this paper, we apply the distributed control methods to solve the real-time economic operation problems in a fully distributed manner. To the best of our knowledge, there is very little work addressing the distributed cooperative control-based economic operation of multiple PEV charging loads from the parking deck operators' perspectives, while considering the inter-relationships among PEV charging facility operators, customers, and utilities.

The major technical contributions of this paper are summarized as follows:

1. To propose a fully distributed methodology and framework to achieve the economic operation of multiple PEV parking decks. 
2. To formulate a global optimization problem to increase the total revenue (i.e., charging revenue and parking revenue) of multiple PEV parking decks while satisfying customer preference (e.g., desired departure battery state-of-charge) and various global and local constraints.

3. To apply consensus algorithm-based distributed control approaches to solve the proposed optimization problem.

4. To evaluate the proposed distributed cooperative control algorithm performance in terms of accuracy, robustness, effectiveness, and scalability.

The remainder of this paper are organized as follows. Section 2 presents the mathematical models of the proposed optimization problem of a number of PEV parking decks. Section 3 introduces the distributed cooperative solution algorithm (consensus-based algorithm). Section 4 discusses the simulation results and the control algorithm performances. Section 5 summarizes the major research findings of this paper and discusses future research trends.

\section{Materials and Methods}

\subsection{Objective functions}

In this paper, a distributed approach is proposed to facilitate the ownership/operation of multiple parking decks to construct the optimal operation strategy in a timely manner. In this approach, the owner/operator purchases electricity from the utility or aggregator. We will be able to determine the optimal charging power allocation for each parking deck in a fully distributed fashion, in response to the dynamic electricity rate as well as power supply. Moreover, we will introduce additional local constraints in order to satisfy customer preferences (e.g., desired battery state-of-charge upon 
departure) and the power constraint of the charging facility (e.g., maximum charging rate in $\mathrm{kW}$ ).

Here, we assume a scenario that multiple parking decks belong to one owner. Therefore, the overall objective is to maximize the total revenue of multiple PEV parking decks in a real-time manner, as expressed as follows:

$$
\operatorname{Max} J_{\text {total }}(t)=\sum_{i=1}^{N} J_{i}(t)
$$

Where $N$ denotes the number of parking decks, $J_{i}$ is the revenue function for the $i$-th parking deck at the $t$-th time slot.

For each parking deck, the revenue function is formulated in Equation (2):

$$
J_{i}(t)=R_{\text {parking }, i}(t)+R_{\text {charging }, i}(t)-C_{\text {operation }, i}
$$

Where $C_{i}$ denotes the fixed operation cost at the $i$-th parking deck, and $R_{\text {parking, } i}(t)$ and $R_{\text {charging, } i}(t)$ are the total parking and PEV charging revenues (\$) of the $i$-th parking deck at the $t$-th time slot, respectively.

The total PEV charging revenue is defined as

$$
R_{\text {charging }, i}(t)=P_{i}(t)\left(r_{\text {charging }, i}-c_{i}(t)\right) \Delta t
$$

Where $P_{i}(t)$ is the total charging power $(\mathrm{kW})$ allocated to the $i$-th parking deck at the $t$-th time step, and $\Delta t$ is the time step. In this paper, we consider a scenario where the parking deck offers a flat electricity sale price to customers. Using flat fee is an efficient way to build confidence relationship between customers and PEV parking decks, since the price for customers is constant during a whole day. $r_{\text {charging, } i}$ denotes the charging fee for all customers at the $i$-th parking deck 
$(\$ / \mathrm{kWh})$. For the sake of simplicity, $r_{\text {charging, } i}$ can be predetermined by day-ahead scheduling, as detailed in our previous work [14]. $c_{i}(t)$ denotes the electricity purchase charge $(\$ / \mathrm{kWh})$ for the $i$-th parking deck at the $t$-th time step from the utility or aggregator.

The high power demand of each parking deck will impact the power loss and the transmission congestion. The distribution locational marginal pricing (DLMP) concept [23], [24] has been successfully developed and implemented in many real-world applications to mitigate the congestion issue with EV charging. Therefore, we consider a distribution locational marginal pricing-like (DLMP-like) method to consider the distribution line congestion caused by the PEV charging loads. Accordingly, the prices vary with location. DLMP is a mechanism for using market-based prices for managing distribution congestion. The power consumption of any selected parking deck may have noticeable impact on the distribution line congestion. The impact will be even exaggerated when the parking deck is connected to a node/bus with heavy base load. Here, we use a simplified demand response function to mimic the DLMP-like electricity price. The electricity purchase price for the $i$-th parking deck, offered by the utility or aggregator at each time slot $t$, is formulated as below:

$$
c_{i}(t)=\alpha_{i} \cdot P_{i}(t)+\beta_{i}
$$

Where $\alpha_{i}$ and $\beta_{i}$ are load-demand curve coefficients.

In addition, the total parking revenue for the $i$-th parking deck is expressed in Equation (5).

$$
R_{\text {parking }, i}(t)=M_{i}(t)\left(1-\rho_{i}(t)\right) r_{\text {parking }, i} \Delta t
$$

$M_{i}(t)$ is the number of PEVs being charged in the $i$-th parking deck at the $t$-th time slot. $r_{\text {parking, } i}$ 
is the parking fee (\$/hour) for all vehicles in the $i$-th parking deck at the $t$-th time interval. $\rho_{i}(t)$ is the parking fee rebate rate between $0 \%$ and $100 \%$. For example, when the parking fee rebate rate is $30 \%$, customers will receive $30 \%$ discount on their parking fee when their PEVs are being recharged. The main purpose of setting rebate rate is to encourage customer to recharge their PEVs at the parking deck instead of parking only. The rebate rate function at the $i$-th parking deck is set to be inversely proportional to the aggregate power consumption. $\rho_{i}(t)$ is formulated in Equation (6).

$$
\rho_{i}(t)=\left\{\begin{array}{lr}
\gamma_{i}-\theta_{i} P_{i}(t) & \text { if } P_{i}<\frac{\gamma_{i}}{\theta_{i}}, \\
0 & \text { if } P_{i} \geq \frac{\gamma_{i}}{\theta_{i}},
\end{array}\right.
$$

Where $\theta_{i}$ and $\gamma_{i}$ are pre-determined parameters, which are related to the parking deck owner's estimation for the present market. Each parking deck can have its own rebate by selecting coefficients $\theta_{i}$ and $\gamma_{i}$, but all parking decks should limit their rebate rate within the reference $(0 \%-100 \%)$

\subsection{Global constraint}

The aforementioned optimization problem is under the power balance equation and local load constraints for each parking deck. For any given time slot $t$, the real power balance equation is given in Equation (7).

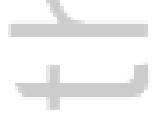

$$
\sum_{i=1}^{N} P_{i}(t)-P_{\text {grid }}(t)=0
$$

Where $P_{\text {grid }}(t)$ is the power supply from the power provider at the $t$-th time step in $\mathrm{kW}$. For the sake of simplicity, power loss is neglected in this paper. The total charging power of all parking 
decks is equal to the power from the utility grid.

\subsection{Local constraint}

Each parking deck is also subject to local power limits:

$$
\forall i \in N: 0 \leq P_{i}(t) \leq P_{i, \max }
$$

Where $P_{i, \max }$ is the maximum total charging power allocated to the $i$-th parking deck in $\mathrm{kW}$. Additionally, in order to ensure that each customer of the charging stations can achieve their expected battery state-of-charge (SOC) at departure, we need to consider the minimal required total charging power, $P_{i, \min }(t)$, for the $i$-th parking deck at the $t$-th time slot in $\mathrm{kW}$.

$$
\forall i \in N: P_{i}(t) \geq P_{i, \min }(t)
$$

Where the predicated required charging power $P_{i, \min }(t)$ is defined as the total of the predicated average charging power for each PEV at time slot $t$.

$$
\forall i \in N, q \in M_{i}: P_{i, \min }(t)=\omega_{i} \sum_{q=1}^{M_{i}} P_{i, q}(t)
$$

Where $\omega_{i}(0 \sim 1)$ is a relaxation parameter and varies in different parking decks. Each parking deck owner has to take the risk of energy transaction with the utility grid, which could be caused by the inaccurate prediction. $\omega_{i}$ is used to reflect the risk tolerance of the $i$-th parking deck owner. The greater the relaxation parameter is, the higher risk the parking deck owner is willing to take.

Considering the uncertainties of different PEV customers' behaviors in different parking decks, we define the predicated average charging power for the $q$-th PEV in the $i$-th parking deck at time slot $t$ as: 


$$
\forall i \in N, q \in M_{i}: P_{i, q}(t)=\frac{\left[\operatorname{SOC}_{i, q}(\text { end })-S O C_{i, q}(t)\right] \operatorname{Cap}_{i, q}}{\left(T_{o u t, i, q}-t\right) \cdot \Delta t}
$$

Where $S O C_{i, q}($ end) denotes the objective SOC of user $q$ in the $i$-th parking deck, which is determined by the user. $S O C_{i, q}(t)$ is the present $S O C$ for the $q$-th PEV in the $i$-th parking deck at the $t$-th time slot. $T_{\text {out }, i, q}$ is the preset departure time for the $q$-th PEV in the $i$-th parking deck. $\operatorname{Cap}_{i, q}$ is the capacity of the $q$-th battery in the $i$-th parking deck in Columbs. Here, $P_{i, q}(t)$ is a predicated value. It is worth to mention that this paper is more focused on the estimated amount of power allocation for a parking deck instead of a single PEV. After allocating the power to each parking deck, we then distribute the allocated power to each PEV considering its maximum charging power constraint.

Therefore, the original optimization problem in Equation (1) can be reformulated as follows:

$$
\begin{array}{r}
\text { Min }-J_{\text {total }}(t)=\sum_{i=1}^{N}\left(-J_{i}(t)\right) \\
\text { s.t. } \sum_{i=1}^{N} P_{i}(t)-P_{\text {grid }}(t)=0 \\
\forall i \in N: P_{i, \min }(t)<P_{i}(t)<P_{i, \max }
\end{array}
$$

\subsection{Centralized solution}

Similar to a traditional economic dispatch (ED) problem, we can apply the Lagrange relaxation method to solve (12) in a centralized manner. The key to solve an ED problem is the incremental cost, which is the cost to generate the next unit amount of power. Here, the incremental cost is the first-order derivative of the cost function with respect to the power output. Applying the Lagrange 
method to the objective function, we get a set of equations:

$$
\frac{\partial \mathcal{L}}{\partial P_{i}}=\frac{\partial\left(-J_{i}\right)}{\partial P_{i}}-\lambda_{i}=0
$$

Where $\mathcal{L}$ is the Lagrange operator. Equivalently, we get the incremental cost:

$$
\lambda_{i}=\frac{\partial\left(-J_{i}\right)}{\partial P_{i}}=2 \Delta t \cdot \alpha_{i} \cdot P_{i}(t)+\Delta t\left\{\beta_{i}-r_{\text {charging }, i}-\theta_{i} r_{\text {parking, } i} M_{i}(t)\right\}
$$

To get the minimum value of $-J_{\text {total }}$, all incremental costs should be equal to the same $\lambda^{*}$. Since $\frac{\partial^{2}\left(-J_{i}\right)}{\partial P_{i}{ }^{2}}=2 \Delta t \cdot \alpha_{i}>0$, the objective function is convex. The optimal solution for such a concave/convex problem is given by [25]:

$$
\begin{gathered}
\lambda^{*}=\frac{\left(-P_{\text {grid }}(t)+\sum_{i}^{N} \frac{\beta_{\mathrm{i}}-\mathrm{r}_{\text {charging, } \mathrm{i}}-M_{i}(t) \theta_{i} r_{\text {parking }, i}}{2 \alpha_{i}}\right)}{\left(\sum_{i}^{N}-\frac{1}{2 \Delta \mathrm{t} \alpha_{i}}\right)} \\
P_{i}^{*}(\mathrm{t})=\frac{\Delta \mathrm{t}\left(\beta_{\mathrm{i}}-\mathrm{r}_{\text {charging, } \mathrm{i}}-M_{i}(t) \theta_{i} r_{\text {parking }, i}\right)-\lambda^{*}}{-2 \Delta \mathrm{t} \alpha_{i}}
\end{gathered}
$$

When considering the constraints, the well-known solution is

$$
\left\{\begin{array}{cc}
\lambda^{*} \leq \frac{\partial\left(-J_{i}\right)}{\partial P_{i}} & P_{i}(t)=P_{i, \min } \\
\lambda^{*}=\frac{\partial\left(-J_{i}\right)}{\partial P_{i}} & P_{i, \min }(t) \leq P_{i}(t) \leq P_{i, \max } \\
\lambda^{*} \geq \frac{\partial\left(-J_{i}\right)}{\partial P_{i}} & P_{i}(t)=P_{i, \max }
\end{array}\right.
$$

Obviously, the centralized method is based on the assumption that the central controller has a full access to all needed information from all local agents through a dedicated communication network. Additionally, each participant needs access to the global information.

\section{Theory}

\subsection{Graph Theory}


An undirected graph $G$ is used here to model the interaction topology of a network of agents. Denote $G=(V, E)$ as a graph with a set of vertices $V=\{1,2, \ldots, n\}$ and edges $E \subseteq V \times$ $V$. An undirected edge $(i, j)$ denotes that agents $i$ and $j$ can obtain information from each other. The set of neighbors of agent $i$ is denoted by $\left.N_{i}=\{j \in V \mid(i, j) \in E)\right\}$. Two vertices are called connected if there is a distinct path from agent $i$ to agent $j$. An undirected graph is connected if there exists an undirected path between any pair of vertices. More specifically, an undirected graph is considered as fully connected if there exists an undirected path between every pair of vertices.

The adjacency matrix $D=\left[d_{i j}\right] \in R^{n \times n}$ of an undirected graph $G$ is symmetric, which means $(i, j) \in E$ if and only if $(j, i) \in E$ [31]. Furthermore, the entry $d_{i j}$ of the adjacency matrix is a positive weight if $(i, j) \in E$ and $d_{i j}=0$ if $(i, j) \notin E$. For a continuous-time system, the second smallest eigenvalue of the corresponding Laplace matrix is called its algebraic connectivity. According to [32], the algebraic connectivity of the interaction topology quantifies the speed of convergence of the consensus algorithms.

\subsection{Consensus Algorithm}

In the consensus problem, all agents aim to reach a consensus. In other words, each agent's state is driven toward the state of its neighbors [33]. Regarding each agent in a graph $G$ with single-integrator dynamic $\dot{x}_{i}=u_{i}$, where $u_{i}$ represents the state variable, as in [32], a continuous-time consensus algorithm is given as:

$$
\dot{x}_{i}=u_{i}=\sum_{j=1}^{N} d_{i j}\left(x_{i}-x_{j}\right)
$$


Where $d_{i j}$ is the entry of the adjacency matrix.

The discrete-time consensus algorithm is described as

$$
x_{i}[k+1]=\sum_{j=1}^{N} d_{i j} x_{j}[k]
$$

where $x_{i}[k+1]$ is the updated state of $x_{i}[k]$ at the iteration $k+1, x_{j}[k]$ is the local information discovered by agent $j$ at iteration $k$, and $d_{i j}$ is the entry of the adjacency matrix.

Similar to [25], [26] and [31], the elements of the adjacency matrix for a fully connected graph $G$ are defined as:

$$
d_{i, j}=\left\{\begin{array}{cc}
\frac{1}{\left|N_{i}\right|}, & j \in N_{i} \\
0, & j \notin N_{i}
\end{array}\right.
$$

Where $N_{i}$ is the set of neighbors of vertex $i$, and $|\cdot|$ is its cardinality. Here, since agent $i$ can obtain its own information, $N_{i}$ includes itself.

\subsection{Cooperative control}

In this system, each parking deck is envisioned as an agent. Let $k$ be the iteration index. The consensus algorithms are discussed in [26]. Two consensuses are implemented in parallel, and the incremental cost is updated following the rules below:

$$
\lambda_{i}(k+1)=\sum_{j=1}^{N_{i}} d_{i j} \lambda_{j}(k)+\epsilon \Delta P_{i}(k)
$$

Where $\lambda_{i}(t)$ is the local estimation of the incremental cost for parking deck $i . \Delta P_{i}(k)$ is the local estimation of the power mismatch between the supply and demand and $\epsilon$ is the step size, which is set as 0.00012 in our case study.

According to incremental cost results from (21), the corresponding power allocation for the $i$-th 
parking deck can be calculated locally:

$$
P_{i}(k+1)=\frac{\Delta \mathrm{t}\left(\beta_{\mathrm{i}}-\mathrm{r}_{\text {charging, } \mathrm{i}}-M_{i}(t) \theta_{i} r_{\text {parking }, i}\right)-\lambda_{i}(k+1)}{-2 \Delta \mathrm{t} \alpha_{i}}
$$

The estimated power mismatch also reaches a consensus.

$$
\Delta P_{i}(k+1)=\sum_{j=1}^{N_{i}} d_{i j} \Delta P_{i}(k)-\left(P_{i}(k+1)-P_{i}(k)\right)
$$

If $\epsilon$ is small enough, then the distributed algorithm is guaranteed to be stable and all control variables converge to the true values that can be found using the centralized approach, in another word, the optimality is ensured [20],[26]. The algorithm only requires local information exchange between neighbors. Due to the geographical locations for multiple parking decks, a reliable and two-way communication network is needed to enable the successful implementation of the proposed distributed method. Here, both wireless techniques (e.g., Cellular Network) and wired techniques (e.g., Ethernet) are potentially viable solutions.

Regarding the initialization setting, multiple options can be implemented, ranging from a single point setting to a whole networked point setting. The initialization here is set as (24),

$$
\begin{aligned}
& P_{i}(t)=\left\{\begin{array}{c}
P_{i, \max }, \quad \text { if } \Delta P_{i}(0)>P_{i, \max }, \\
\Delta P_{i}(0) \text { if } P_{i, \min }<\Delta P_{i}(0)<P_{i, \max }, \\
P_{i, \text { min }}, \quad \text { if } \Delta P_{i}(0)<P_{i, \min },
\end{array}\right. \\
& \Delta P_{i}(0)=\left\{\begin{array}{l}
P_{\text {grid }}(t), \quad i=1 \\
0, \quad \text { otherwise }
\end{array}\right. \\
& \lambda_{i}(0)=\left\{\begin{array}{c}
\Delta t\left\{\beta_{i}-r_{\text {charging }, i}-\theta_{i} r_{\text {parking }, i} M_{i}(t)\right\}, \quad t=1 \\
\lambda_{i}(t-1), \quad \text { otherwise }
\end{array}\right.
\end{aligned}
$$

Fig. 2 shows the flowchart of the proposed consensus-based distributed optimization approach. At 
the beginning of the operation, each parking deck will set its own electricity sale price for customers, namely, the parking fee and the parking rebate rate. And then we initialize the incremental cost, the power mismatch estimation, and the local charging power reference using (24). Also, each parking deck receives the electricity price information from the utility company. After the initialization, each parking deck begins to communicate with the neighboring decks and exchanges the incremental cost and the power mismatch estimation information. Then, each parking calculates its own incremental cost and power mismatch estimation using (21) and (23). Meanwhile, each parking deck updates its local total charging power reference according to (22). Accordingly, the incremental cost, the power mismatch estimation, and the charging power reference are calculated and updated locally. Finally, the incremental cost and power mismatch estimation are sent out to its neighbors for their iterative calculations. Gradually, the incremental cost and the power mismatch are converging to the optimal charging power for each parking deck at time step $\mathrm{t}$. We the repeat the same procedure for the next time step. 


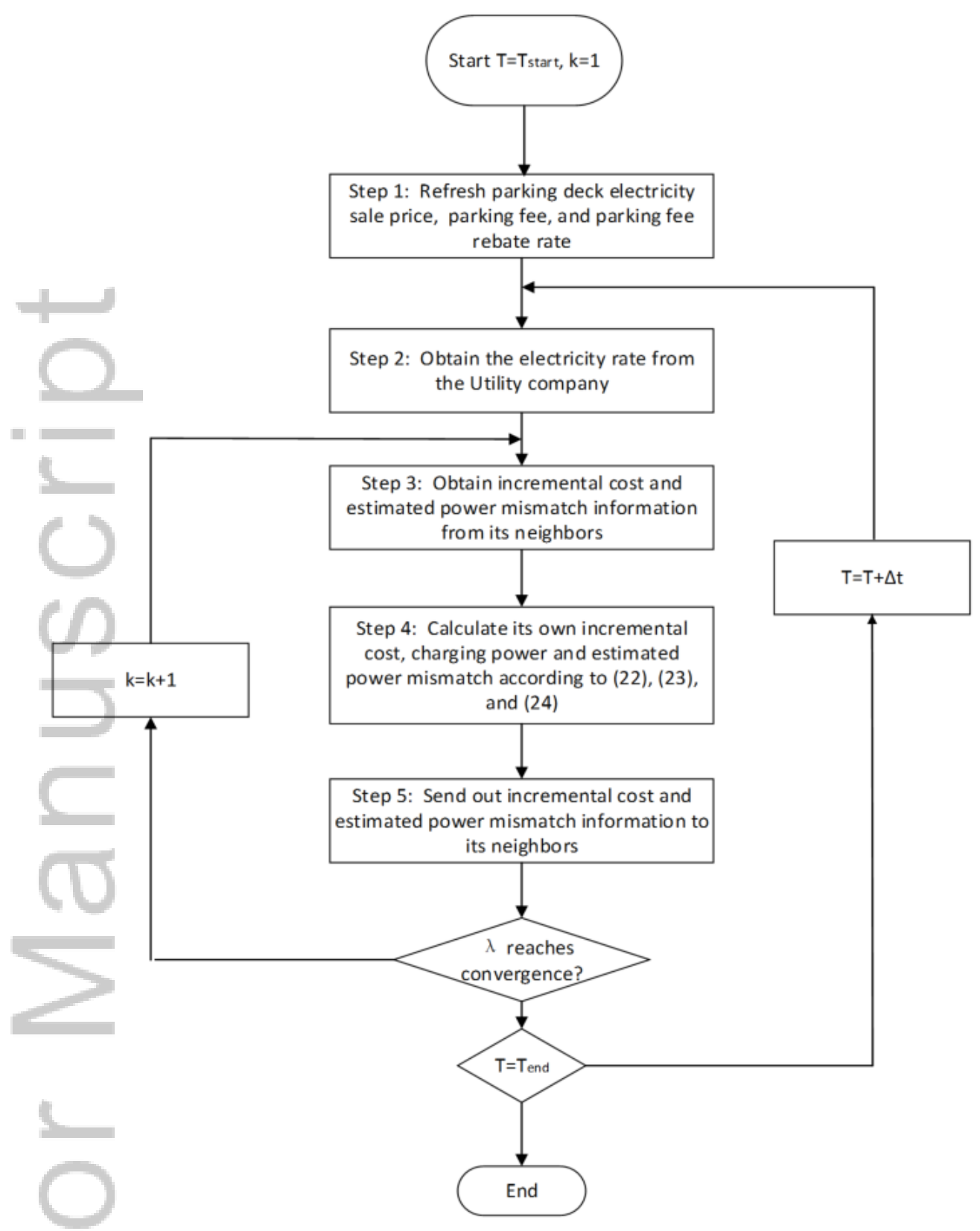

Fig. 2. Flow Chart of Economic Operation Process

The charging strategy of individual PEVs within a parking deck is beyond the scope of this paper.

Therefore, for the sake of simplicity, once the optimal power allocation has been found at each time slot, the real-time charging power for the $k$-th PEV at time slot $t$ will be implemented as: 


$$
P_{i, k}(t)=\frac{P_{i}(t)}{M}
$$

\section{Results and Discussion}

\subsection{System configuration}

This section shows a number of case studies to demonstrate the accuracy, robustness, effectiveness and scalability of the proposed distributed control approach. The first two cases consider a simple 4-parking deck scenario with and without local power constraints. Case study 3 verifies the robustness of the proposed framework under the condition of a time varying power supply form the utility company. Case study 4 investigates the performance of parking decks to satisfy customer preferences. Lastly, the 100-parking deck system in case study 5 is applied to validate the scalability of the proposed framework.

\subsubsection{Case study 1: 4-parking deck system without local constraint}

The 4-parking deck network is fully connected in communication connection. The corresponding communication topology is shown in Fig. 3. The parking fee, charging fee and other initial coefficients are included in Table 2. As for simulations, which have been run in Matlab and Simulink, the step size is $0.1 \mathrm{~s}$; in other words, each agent in our model will communicate with its neighbors every $0.1 \mathrm{~s}$.

This article is protected by copyright. All rights reserved. 


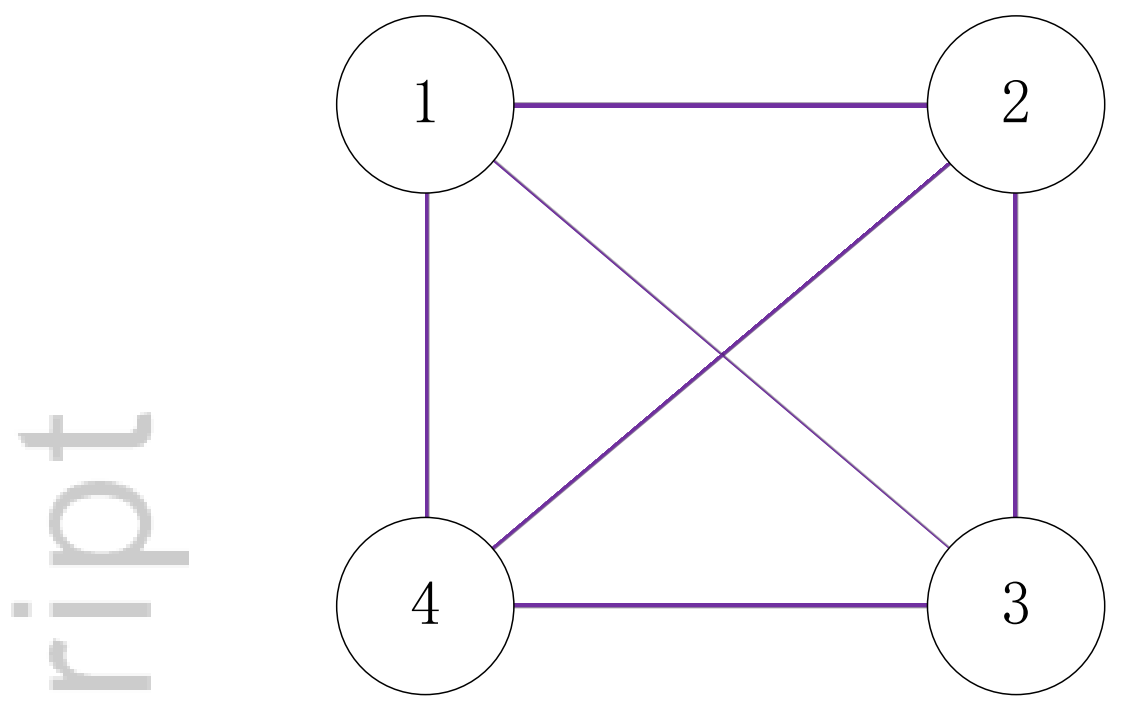

Fig. 3. Communication Topology among 4 Parking Decks

Table 2: Coefficient

\begin{tabular}{cllll}
\hline Parking Decks & $i=1$ & $i=2$ & $i=3$ & $i=4$ \\
\hline $\boldsymbol{\alpha}$ & 0.0025 & 0.0028 & 0.002 & 2.3 \\
\hline $\boldsymbol{\beta}$ & 2.1 & 2.4 & 2 & 3.6 \\
\hline $\mathbf{r}_{\text {charging }}$ & 3.9 & 4.2 & 4.5 & 1.8 \\
\hline $\boldsymbol{r}_{\text {parking }}$ & 1.9 & 2 & 75 & 100 \\
\hline $\boldsymbol{\theta}$ & 35 & 55 & 0.00055 & 0.00065 \\
\hline $\boldsymbol{\gamma}$ & 0.0006 & 0.0005 & 0.85 & 0.75 \\
\hline $\boldsymbol{P}_{\text {min }}$ & 0.8 & 0.9 & 0 & 0 \\
\hline $\boldsymbol{P}_{\text {max }}$ & 269.5 & 0 & 770 & 577.5 \\
\hline
\end{tabular}

In this case study, the local charging power constraints are not being considered. The initial power supply $P_{\text {grid }}$ is $1800 \mathrm{~kW}$, which is considered as constant. The incremental cost, charging power, estimated power mismatch, total power demand and power supply are shown in Fig. 4. Power mismatch between the power supply from the utility and the total of the power demands of all the parking decks converges to zero very fast. The optimal incremental cost converges to $\lambda=$ 
0.045603 within 10 seconds, and the corresponding charging power allocations for the parking decks are found to be: $P_{1}=404.4661 \mathrm{~kW}, P_{2}=363.8233 \mathrm{~kW}, P_{3}=684.0126 \mathrm{~kW}, P_{4}=347.6979 \mathrm{~kW}$. Since its local power constraint is not considered, $P_{1}$ exceeds the maximum charging level and it may overload the charging equipment (e.g., transformer, distribution line).
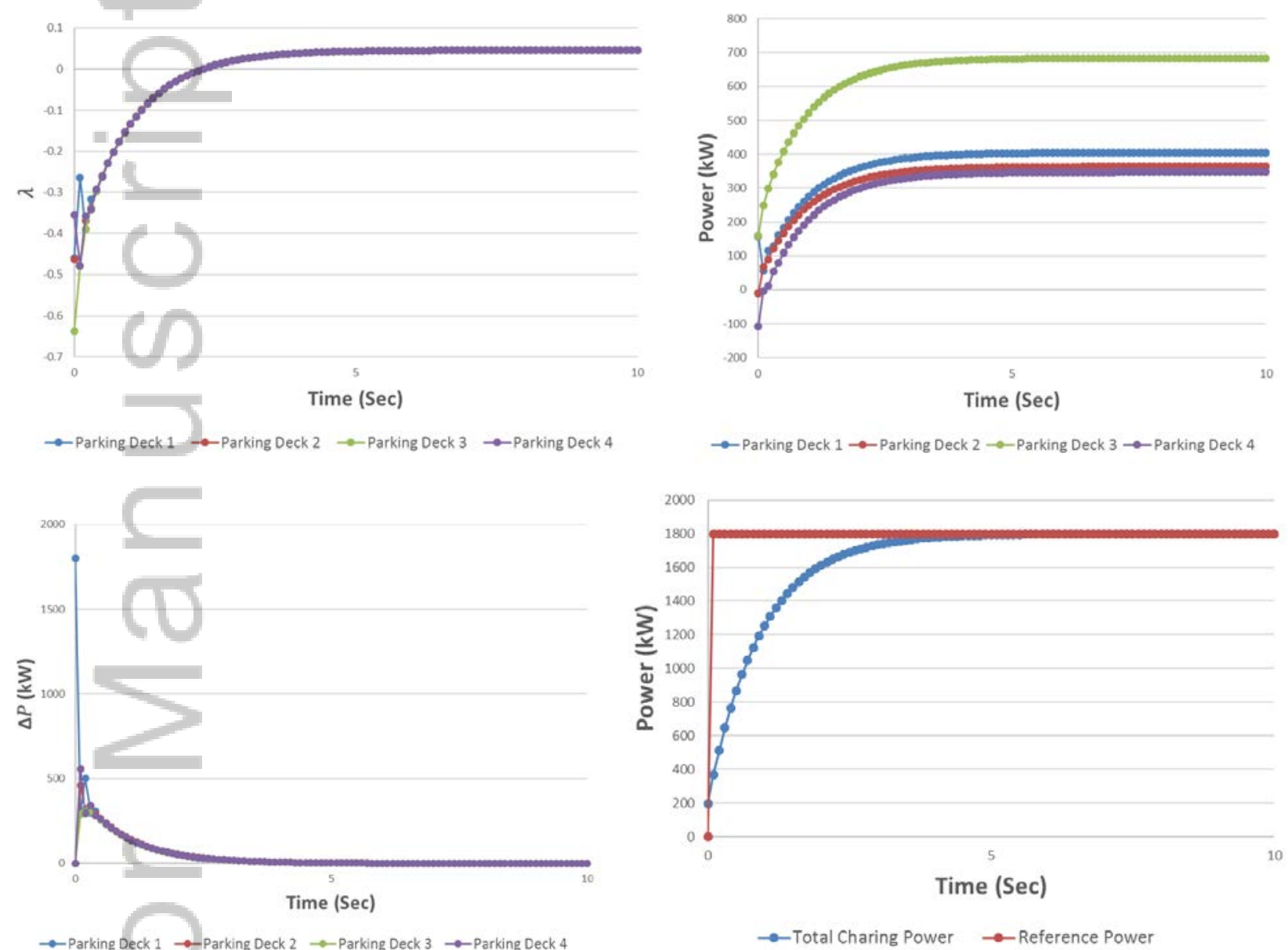

Fig. 4. Test Results for 4-Parking Deck without Local Power Constraint: (a) Incremental Cost, (b) Charging Power, (c) Estimated Power Mismatch, (d) Total Power Demand and Power Supply.

\subsubsection{Case study 2: 4-parking deck system with local constraint}

In case study 2, we impose local constraint on PEV parking deck in order to prove the accuracy of the proposed distributed control approach. All other conditions remain the same as in case study 1. 

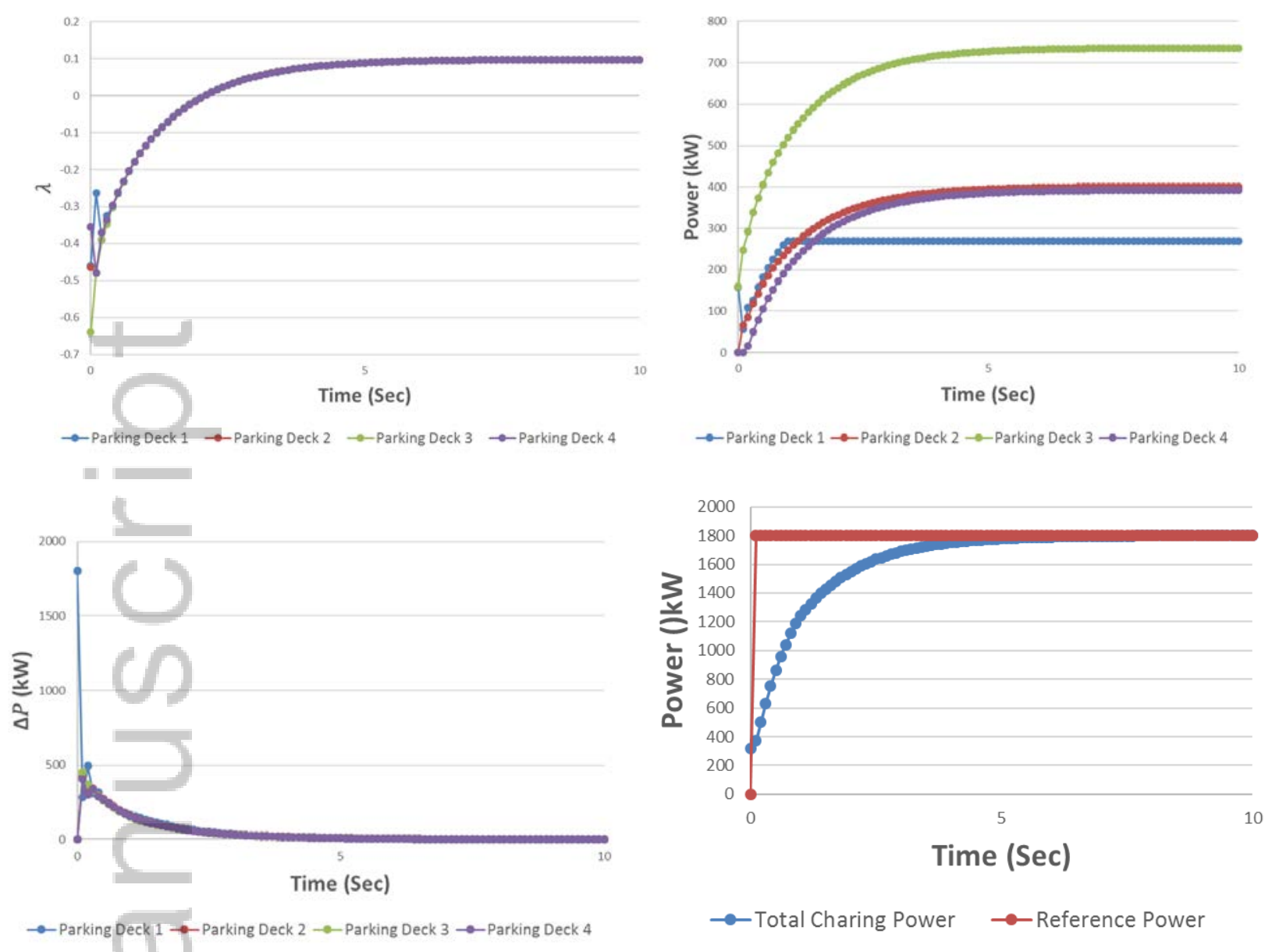

Fig. 5. Test Results for 4-Parking Deck Without Local Power Constraint: (a) Incremental Cost, (b) Charging Power, (c) Estimated Power Mismatch, (d) Total Power Demand and Power Supply. As shown in Fig. 5, $P_{1}$ is limited by its local power constraint. Correspondingly, the optimal incremental cost adjusts to $\lambda=0.09784$, as the final power mismatch still reaches zero. Therefore, the proposed approach can find the optimal power allocation for each parking deck with consideration for their respective local constraints.

\subsubsection{Case study 3: time-varying power supply}

In previous case studies, we assumed that the power supply was constant. In order to prove the robustness of the proposed control approach, a more practical scenario is simulated when imposing 
a time varying power supply. As shown in Fig. 6, since the initial conditions are set to be the same as case study 2, the optimal incremental cost and allocated power are identical in the beginning. At the 25-th second, the power supply changes to $1100 \mathrm{~kW}$. After a very short period, the solution converges again and the power mismatch goes back to zero. The new optimal incremental cost becomes $\lambda=-0.1613$, and the corresponding charging power allocations for the parking decks are found to be: $P_{1}=238.9742 \mathrm{~kW}, P_{2}=216.0627 \mathrm{~kW}, P_{3}=477.1477 \mathrm{~kW}, P_{4}=167.8154 \mathrm{~kW}$. The total charging power is equal to the new power supply.
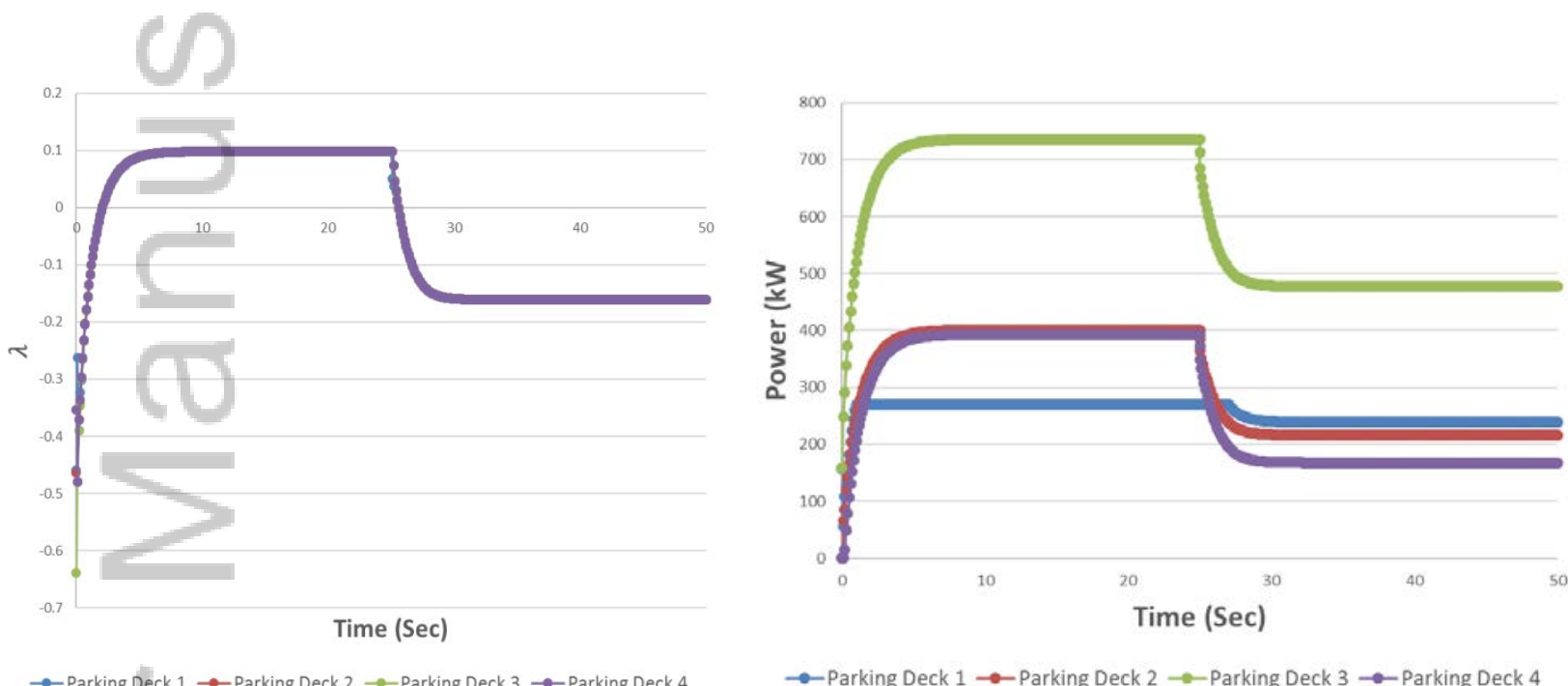

$\rightarrow-$ Parking Deck $1 \rightarrow$ Parking Deck $2 \rightarrow-$ Parking Deck $3 \rightarrow$-Parking Deck 4

$\rightarrow$ Parking Deck $1 \rightarrow$ Parking Deck $2 \rightarrow$ Parking Deck $3 \rightarrow$ Parking Deck 4 


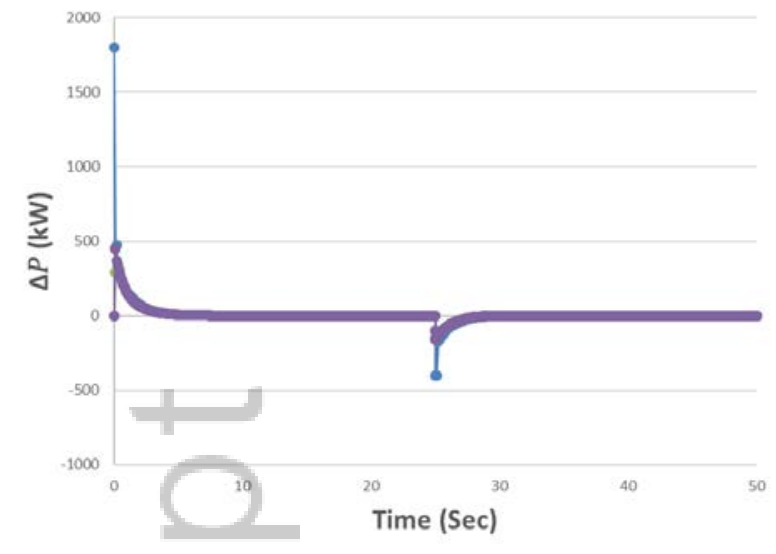

$\rightarrow$ Parking Deck $1 \rightarrow$ Parking Deck $2 \rightarrow$ Parking Deck $3 \rightarrow$ Parking Deck 4

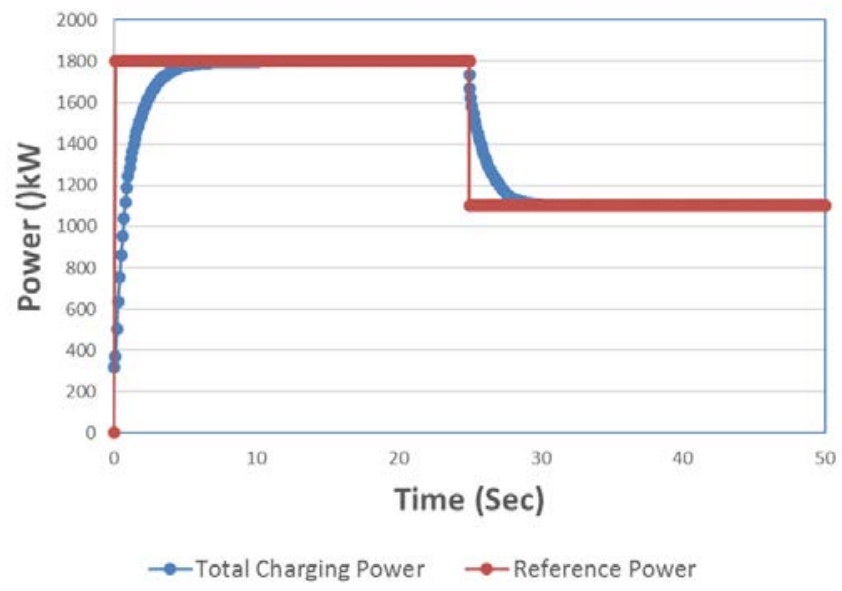

Fig. 6. Test Results for 4-Parking Deck with Local Power Constraint and time varying power supply: (a) Incremental Cost, (b) Charging Power, (c) Estimated Power Mismatch, (d) Total Power Demand and Power Supply.

\subsubsection{Case study 4: desired departure battery SOC}

The effectiveness of the proposed control approach needs to be validated in a real-world PEV charging scenarios. The PEV battery size is set as $24 \mathrm{kWh}$. The PEV chargers are assumed to be AC level 2 (i.e., 240VAC, 32 Amps, and a 7.7 kVA single phase outlet). The initial battery SOC is assumed to follow a Gaussian distribution of $S O C \sim\left(0.6,0.1^{2}\right)$.

In case study 4, we simulate 100 vehicles for each PEV parking deck. All of these vehicles are assumed to plug in at 8:00 AM and depart before 12:00 PM. All customers (i.e., PEV drivers) set their charging goal as $S O C=1$. Fig. 7 shows the simulation results for the four parking decks. Most vehicles reach their preferred battery SOC level. There only exists a very small number of PEVs that are not able to achieve $100 \%$ of their SOC goal. That's because this paper is more focused on the PEV parking deck-level management, thus we implemented a simplified average charging 
method for individual PEVs, as formulated in Equation (25). By incorporating advanced charging control for individual PEVs in the future, we will be able to further improve customer satisfaction in terms of departure battery SOC.
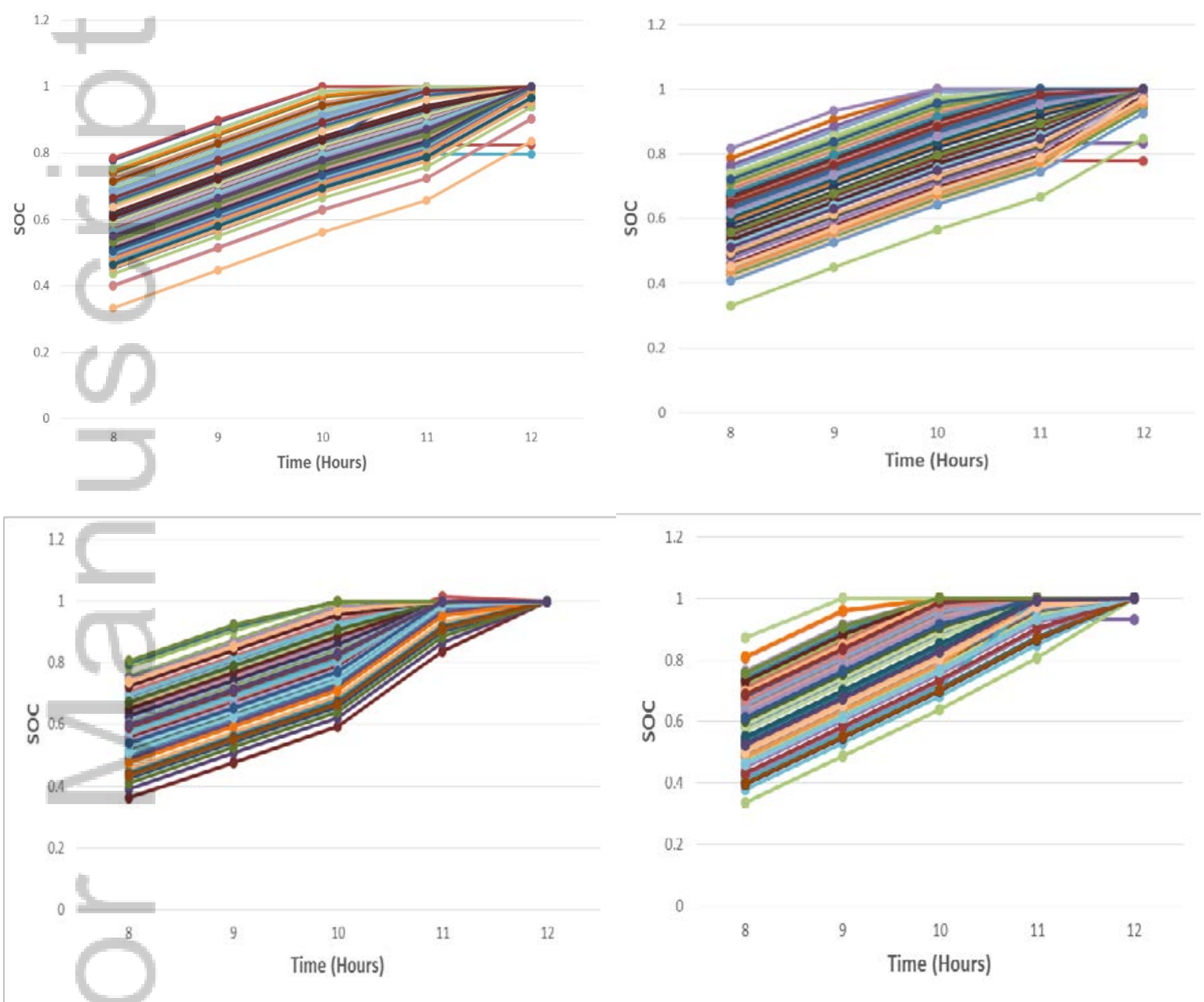

Fig. 7. Case study 4: (a) SOC profiles for PEVs in parking deck 1, (b) SOC profiles for PEVs in parking deck 2, (c) SOC profiles for PEVs in parking deck 3, and (d) SOC profiles for PEVs in parking deck 4

\subsection{5: Case study 5: scalability}

The proposed distributed cooperative control method is suitable for large-scale problem 
optimization in particular. To demonstrate the scalability of the proposed method, we increase the number of PEV parking decks to 100. The initial conditions are similar to those of previous case studies, and the total power supply from the utility is $4000 \mathrm{~kW}$. As shown in Fig. 8, the whole system reaches convergence within approximately 10 seconds, which is considered a reasonable decision making time for 100 PEV parking decks. Besides, in this case, we are using partial connected topology. By increasing the graph density, the convergence speed can be improved at a higher communication cost.

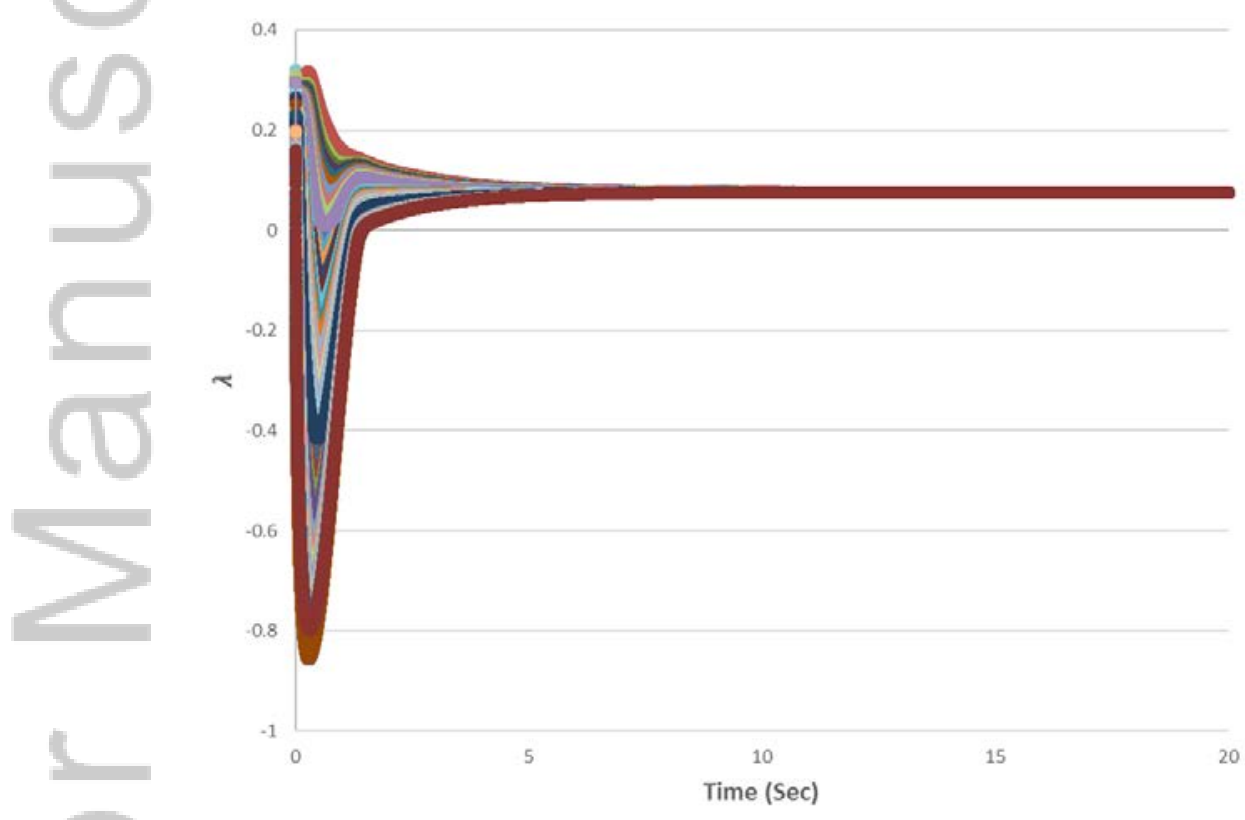

Fig. 8. Test for 100-Parking Deck System

\section{Conclusion}

In this paper, we formulated the economic operation problem to maximize the total parking and PEV charging revenue for the owner of multiple PEV parking decks, with consideration for a number of local constraints (e.g., desired departure battery SOC and maximum charging power 
limit) and global constraints (e.g., power balance). We proposed a distributed framework and applied consensus algorithm-based distributed control algorithms to solve the abovementioned global optimization problem. Each PEV parking deck negotiates with neighboring decks to reach consensus iteratively via two-way communication networks. Due to its lack of a need for heavy information exchange between the master controller and the individual PEV parking decks, distributed control can greatly reduce the computational overhead. We also evaluated the performance of the proposed distributed cooperative control approaches in terms of accuracy, effectiveness, and scalability. In the future, we will further incorporate advanced vehicle-level charging control into the proposed PEV parking deck-level management approaches.

\section{Reference}

[1] C. C. Chan, The state of the art of electric, hybrid, and fuel cell vehicles, Proc. of the IEEE. 95 (2007) 704-718.

[2] M. Sorrentino, G. Rizzo, L. Sorrentino, A study aimed at assessing the potential impact of vehicle electrification on grid infrastructure and road-traffic greenhouse emissions, Applied Energy. 120 (2014) 31-40.

[3] P. J. Tulpule, V. Marano, S. Yurkovich and G. Rizzoni, Economic and environmental impacts of a PV powered workplace parking garage charging station. Applied Energy. 108 (2013) 323-332. 
[4] W. Su, H. Rahimi-Eichi, W. Zeng, and M.-Y. Chow, A survey on the electrification of transportation in a smart grid environment, IEEE Trans. Industrial Informatics. 8 (2012) $1-10$.

[5] W. Su, J. Wang, and Z. Hu, Planning, control and management strategies for parking lots for PEVs, Plug-In Electric Vehicles in Smart Grid: Management and Control Strategies, Edited by S. Rajakaruna, F. Shahnia, and A. Ghosh, Springer, 2014.

[6] Q. Kang, J. Wang, M. Zhou, and A.C. Ammari, "Centralized Charging Strategy and Scheduling Algorithm for Electric Vehicles Under a Battery Swapping Scenario", IEEE Trans. on Intelligent Transportation Systems, vol.17, no.3, pp.659-669, Nov. 2015.

[7] Z. Xu, Z. Hu, Y. Song, W. Zhao and Y. Zhang, Coordination of PEVs charging across multiple aggregators, Applied Energy. 136 (2014) 582-589.

[8] C. Jin, J. Tang, and P. Ghosh, Optimizing electric vehicle charging with energy storage in the electricity market, IEEE Trans. Smart Grid. 4 (2013) 311-320.

[9] O. Sundstrom, and C. Binding, Flexible charging optimization for electric vehicles considering distribution grid constraints, IEEE Trans. Smart Grid. 3 (2012) 26-37.

[10] M.E. Khodayar, L. Wu, and M. Shahidehpour, Hourly coordination of electric vehicle operation and volatile wind power generation in SCUC, IEEE Trans. Smart Grid. 3 (2012) 1271-1279.

[11] T. Sattarpour and M. Farsadi, Parking lot allocation with maximum economic benefit in a distribution network, International Transactions on Electrical Energy Systems. 2016. 
[12] A. Badri, K. H. Lonbar, A short-term optimal decision making framework of an electricity retailer considering optimized EVs charging model, International Transactions on Electrical Energy Systems. 26(2016) 1705-1724.

[13] W. Su, and M.-Y. Chow, Performance evaluation of an EDA-based large-scale plug-in hybrid electric vehicle charging algorithm, IEEE Trans. on Smart Grid. 3 (2012) 308-315.

[14] Y. Guo, J. Xiong, S. Xu, and W. Su, Two-stage economic operation of microgrid-like electric vehicle parking deck, IEEE Trans. on Smart Grid. 2016 (in press).

[15] X. Luo, S. Xia and K. Chan, A simple decentralized charging control scheme of plug-in electric vehicles for alleviating wind farm intermittency, Energy Procedia. 61 (2014) 1789-1792.

[16] Y. He, B. Venkatesh, and L. Guan, Optimal scheduling for charging and discharging of electric vehicles, IEEE Trans. Smart Grid. 3 (2012) 1095-1105.

[17] Y. Cao, S. Tang, C. Li, P. Zhang, Y. Tan, Z. Zhang, and J. Li, An optimized EV charging model considering TOU price and SOC curve, IEEE Trans. Smart Grid. 3 (2012) 388-393.

[18] D'hulst, F. De Ridder, B. Claessens, L. Knapen and D. Janssens, Decentralized coordinated charging of electric vehicles considering locational and temporal flexibility, European Transactions on Electrical Power. 25(2015) 2562-2575.

[19] C.K. Wen, J.C. Chen, J.H. Teng, and P. Ting, Decentralized plug-in electric vehicle charging selection algorithm in power systems, IEEE Trans. Smart Grid. 3 (2012) 1779-1789. 
[20] Y. Xu, Optimal distributed charging rate control of plug-in electric vehicles for demand management, IEEE Trans. Power System. 30 (2015) 1536-1545.

[21] N. Rahbari-Asr and M.-Y. Chow, Cooperative distributed demand management for community charging of PHEV/PEVs based on KKT conditions and consensus networks," IEEE Transactions on Industrial Informatics. 10 (2014) 1907-1916.

[22] J. Miranda, J. Borges, D. Valério, MJGC. Mendes, Multi-agent management system for electric vehicle charging, International Transactions on Electrical Energy Systems. 25(2015) $770-788$.

[23] R. Li, Q. Wu, and S. S. Oren, Distribution Locational Marginal Pricing for Optimal Electric Vehicle Charging Management, IEEE Tran. Power Grid. 29(2014) 203-211.

[24] S. Huang, Q. Wu, S. S. Oren, R. Li, and Z. Liu, Distribution Locational Marginal Pricing Through Quadratic Programming for Congestion Management in Distribution Networks, IEEE Tran. Power Grid. 30(2015) 2170-2178.

[25] G. Binetti, A. Davoudi, F. L. Lewis, D. Naso and B. Turchiano, Distributed consensus-based economic dispatch with transmission losses, IEEE Trans. Power System. 29 (2014) 1711-1720.

[26] S. Yang, S. Tan, and J. Xu, Consensus based approach for economic dispatch problem in a smart grid, IEEE Trans. Power System. 28 (2013) 4416-4426.

[27] Y. Xu, W. Zhang, G. Hug, S. Kar and Z. Li, Cooperative control of distributed energy storage systems in a microgrid, IEEE Trans. Smart Grid. 6 (2015) 238-248. 
[28] Z. Fan, A distributed demand response algorithm and its application to PHEV charging in smart grids, IEEE Trans. Smart Grid. 3 (2012) 1280-1290.

[29] F. Kelly, A. Maulloo, and D. Tan, Rate control for communication networks: shadow prices, proportional fairness and stability, J. Oper. Res. Soc., 49 (1998) 237-252.

[30] Z. Zhang and M. Chow, Convergence analysis of the incremental cost consensus algorithm under different communication network topologies in a smart grid, IEEE. Trans. Smart Grid. 27 (2012) 1761- 1768.

[31] A. Olshevsky and J. N. Tsitsiklis, Convergence speed in distributed consensus and averaging, SIAM, J. Control Optim., 48 (2009) 33-55.

[32] R. Olfati-Saber, J. A. Fax Fax and R. M. Murray, Consensus and cooperation in networked multi-agent systems, Proc. of the IEEE, 95 (2007) 215-233.

[33] W. Ren and Y. Cao, Distributed coordination of multi-agent networks, London, U.K.: Springer, 2011.

[34] W. Su, J. Wang and D. Ton, Smart Grid Impact on Operation and Planning of Electric Energy Systems, Handbook of Clean Energy Systems, Edited by A. Conejo and J. Yan, Wiley, 2015.

This article is protected by copyright. All rights reserved. 


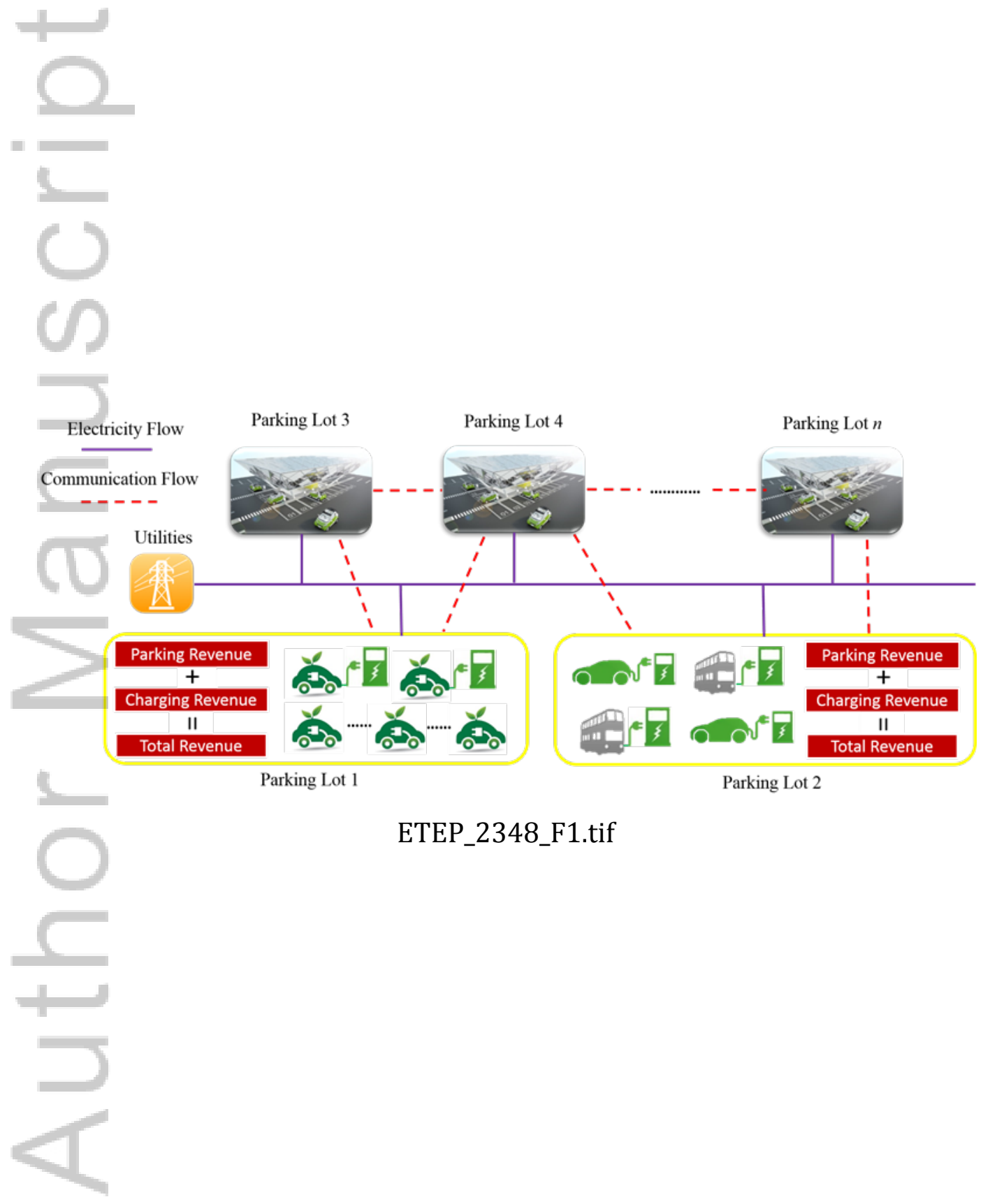

This article is protected by copyright. All rights reserved. 


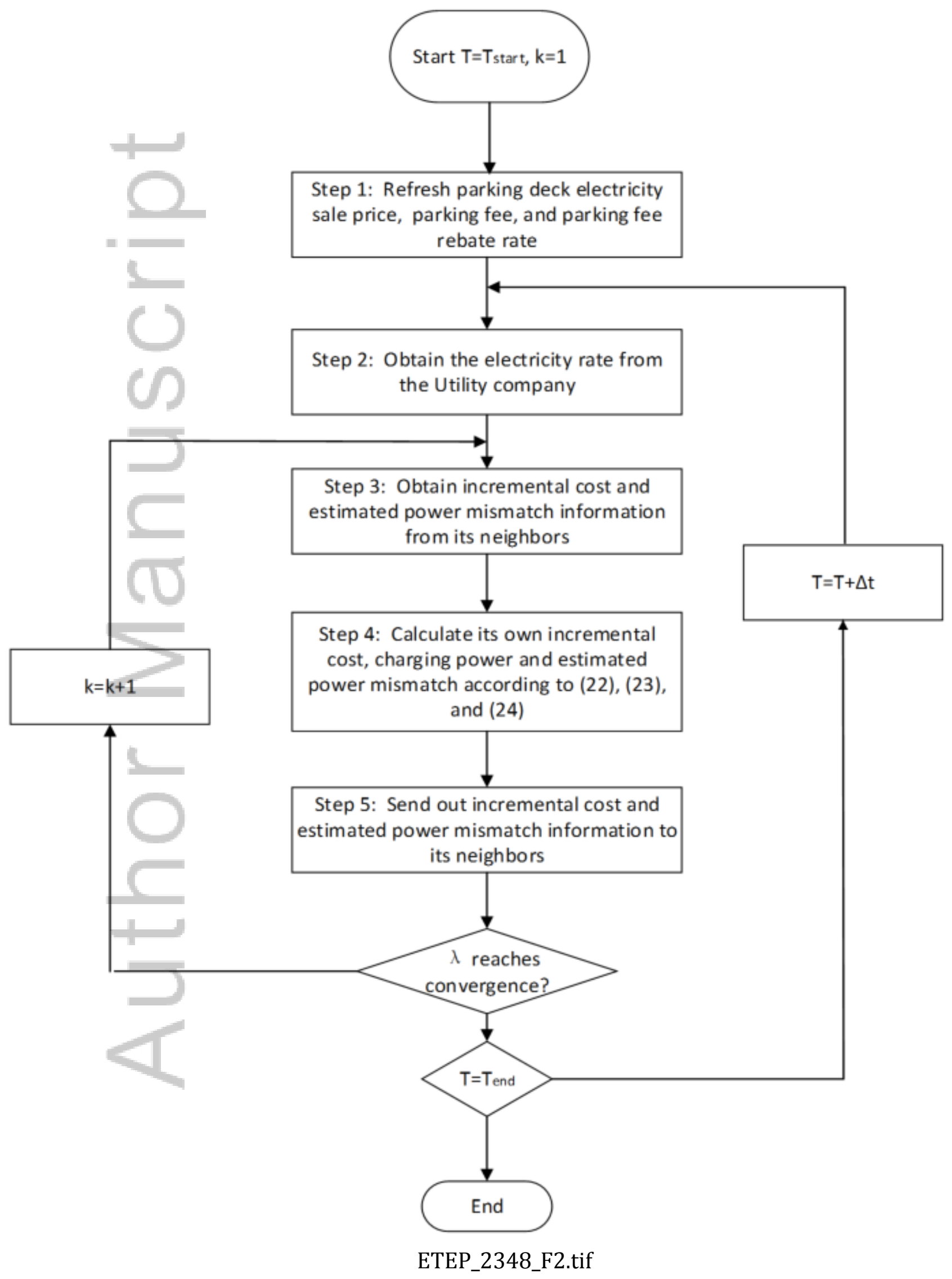

This article is protected by copyright. All rights reserved. 


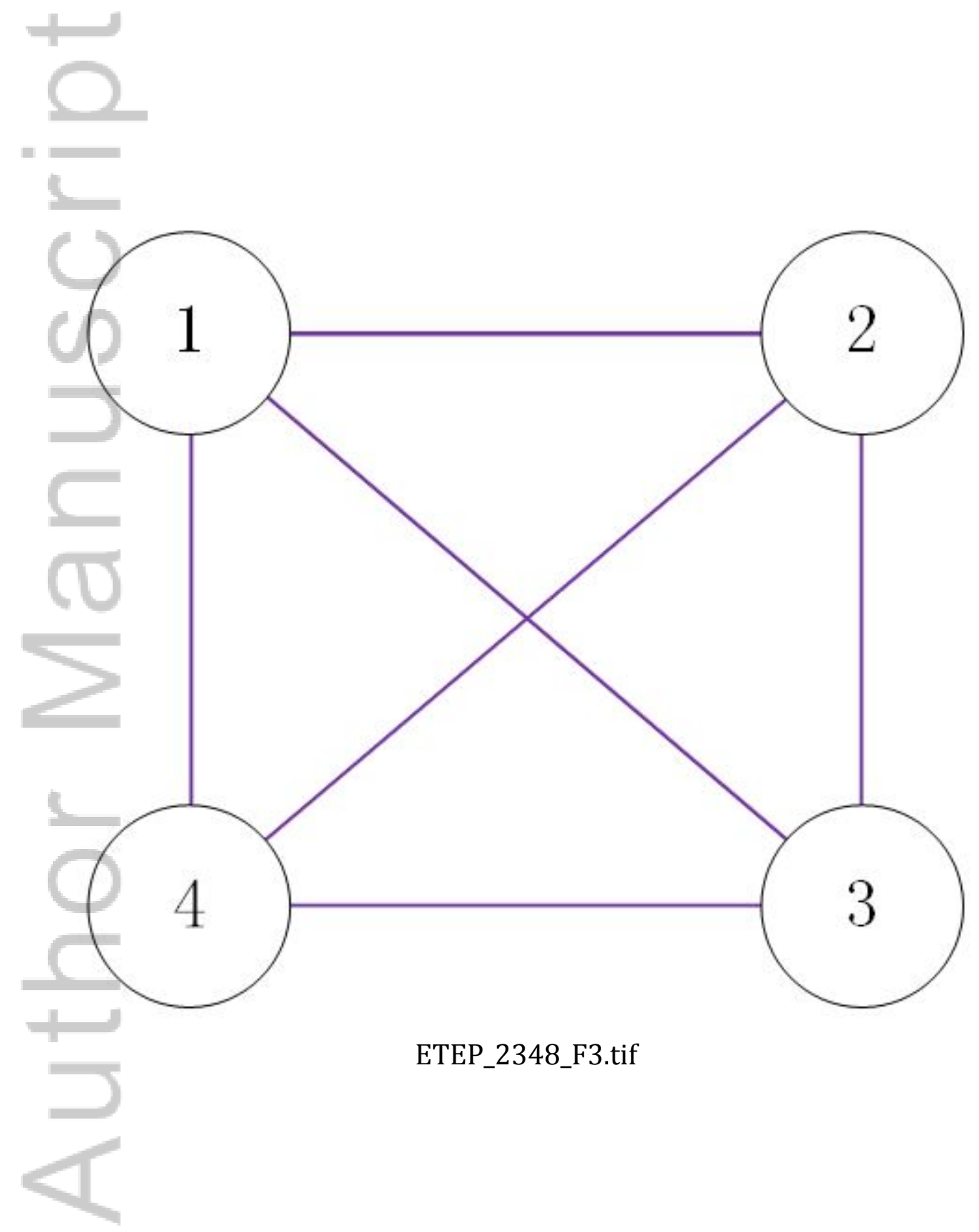

This article is protected by copyright. All rights reserved. 


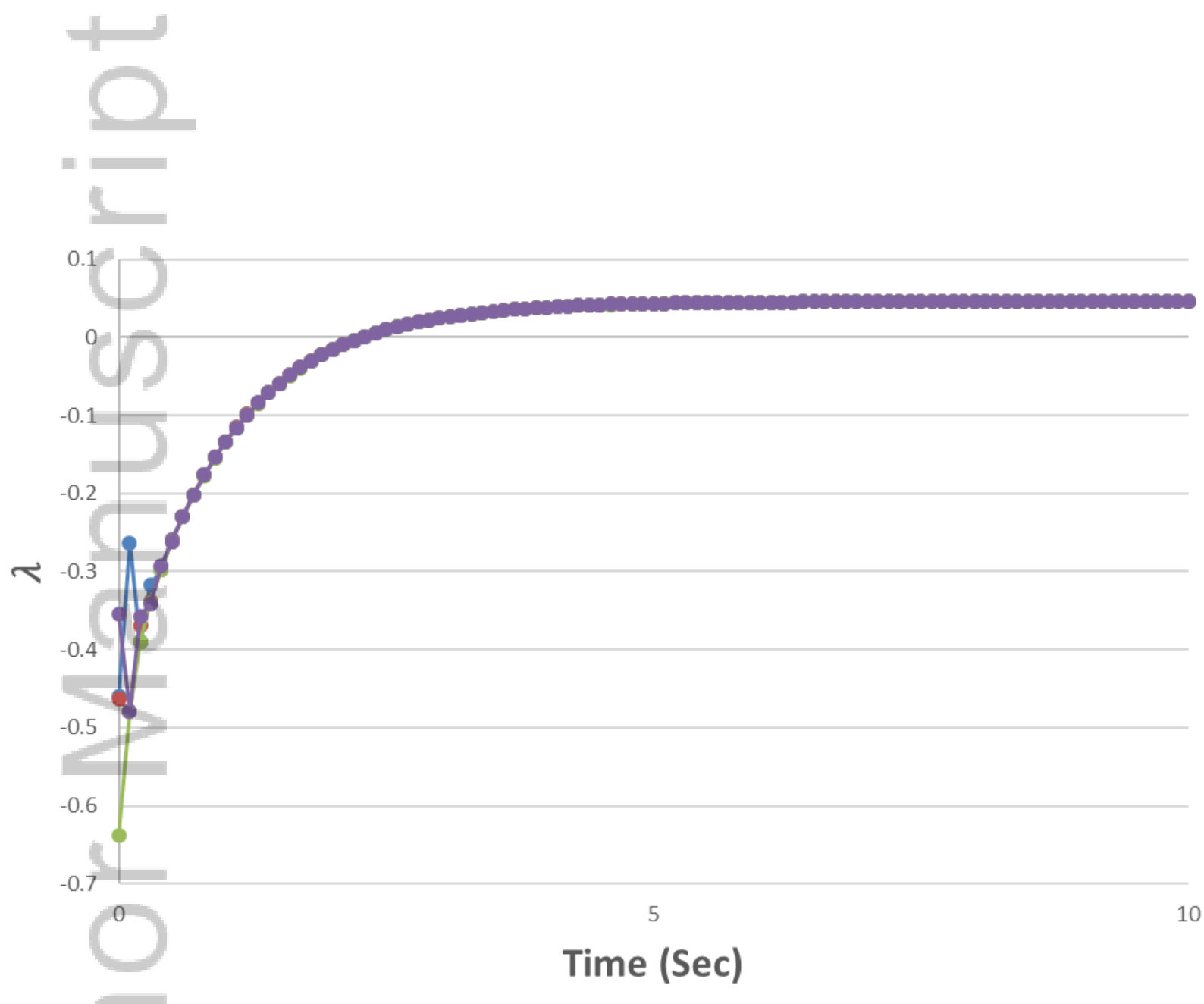

$\multimap$ Parking Deck $1 \rightarrow$ Parking Deck $2 \multimap$ Parking Deck $3 \rightarrow$ Parking Deck 4 $+1$

ETEP_2348_F4(a).tif

This article is protected by copyright. All rights reserved. 

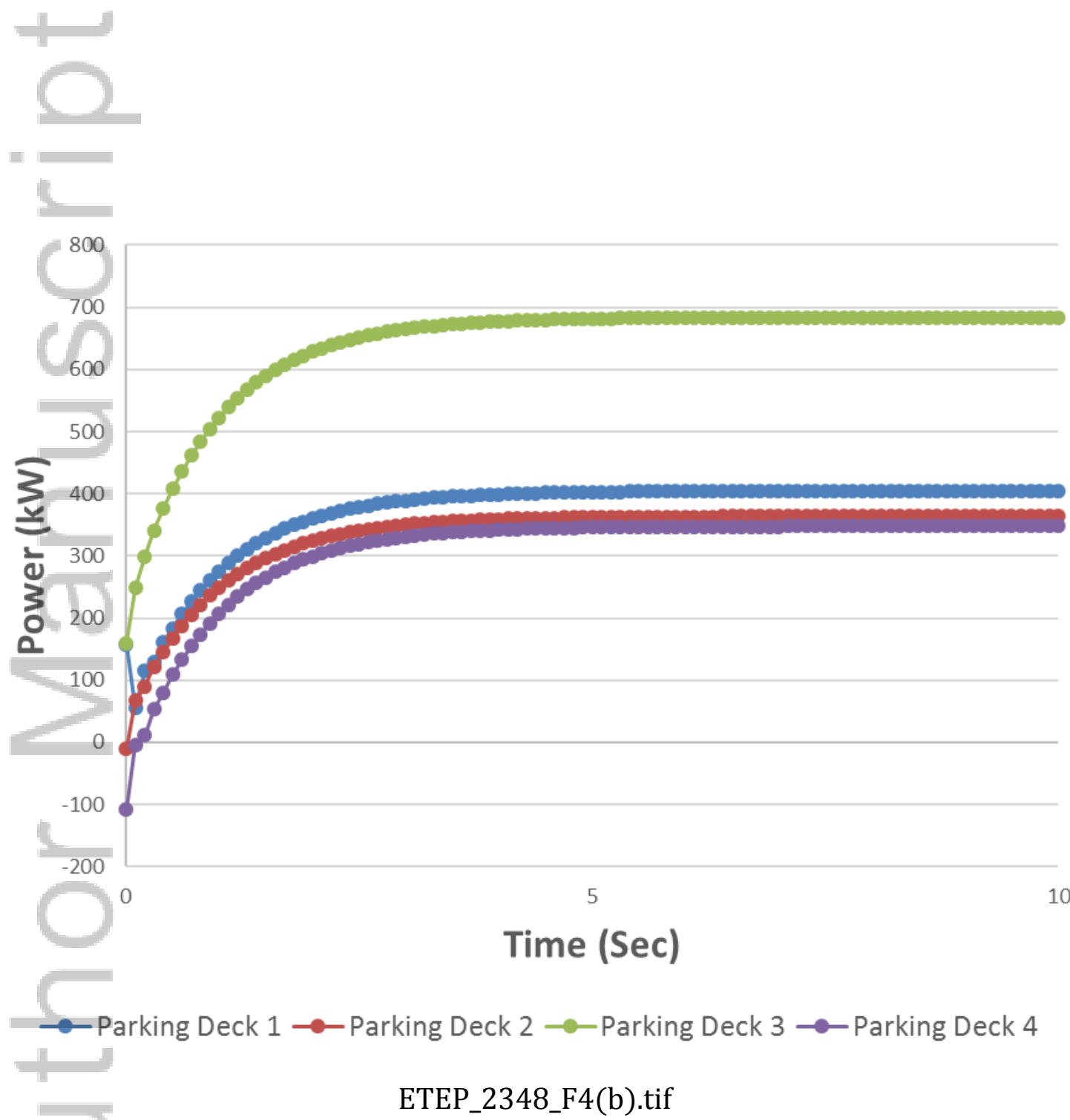

This article is protected by copyright. All rights reserved. 


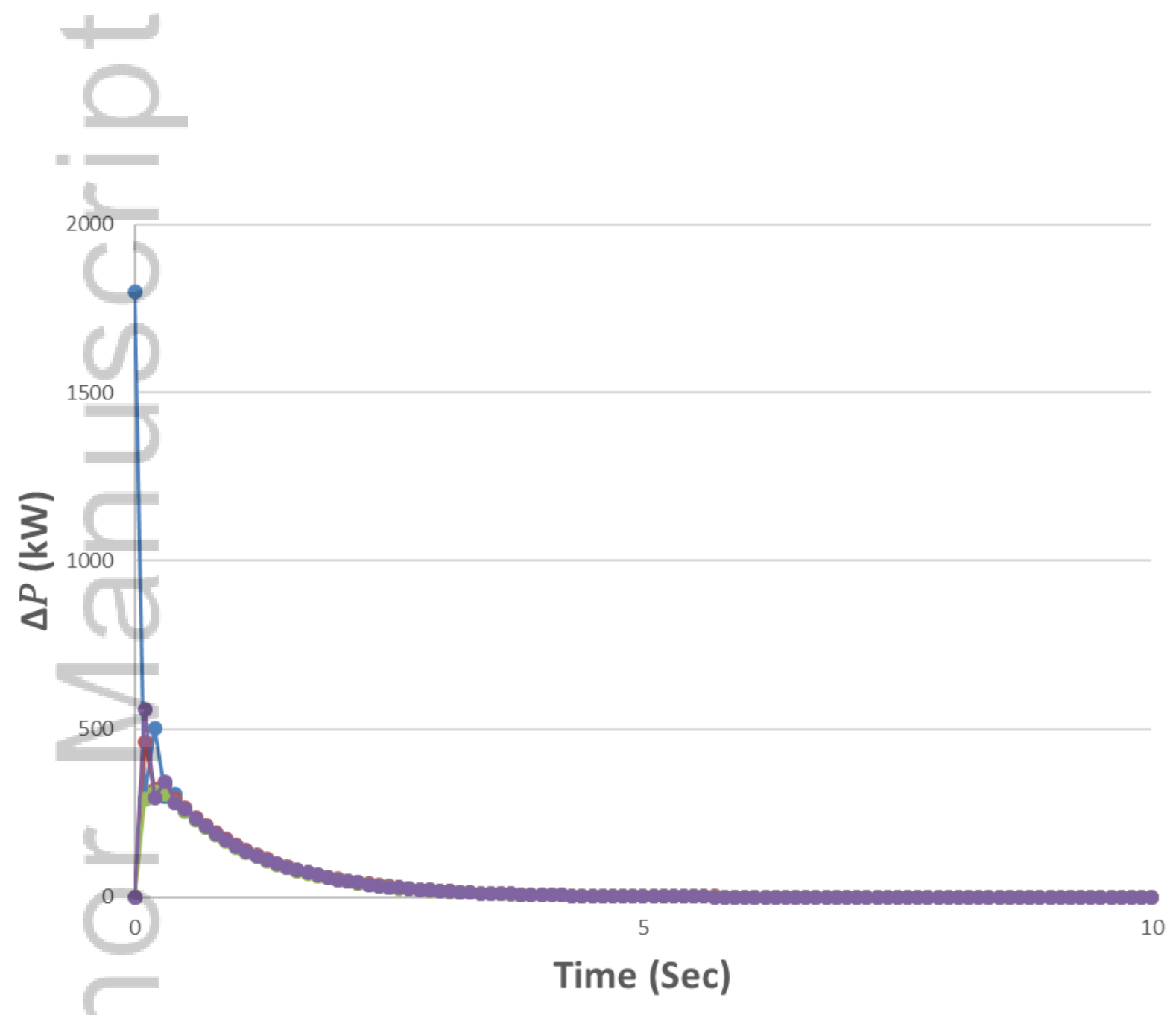

$\multimap$ Parking Deck $1 \multimap$ Parking Deck $2 \multimap$ Parking Deck $3 \multimap$ Parking Deck 4 ETEP_2348_F4(c).tif

This article is protected by copyright. All rights reserved. 


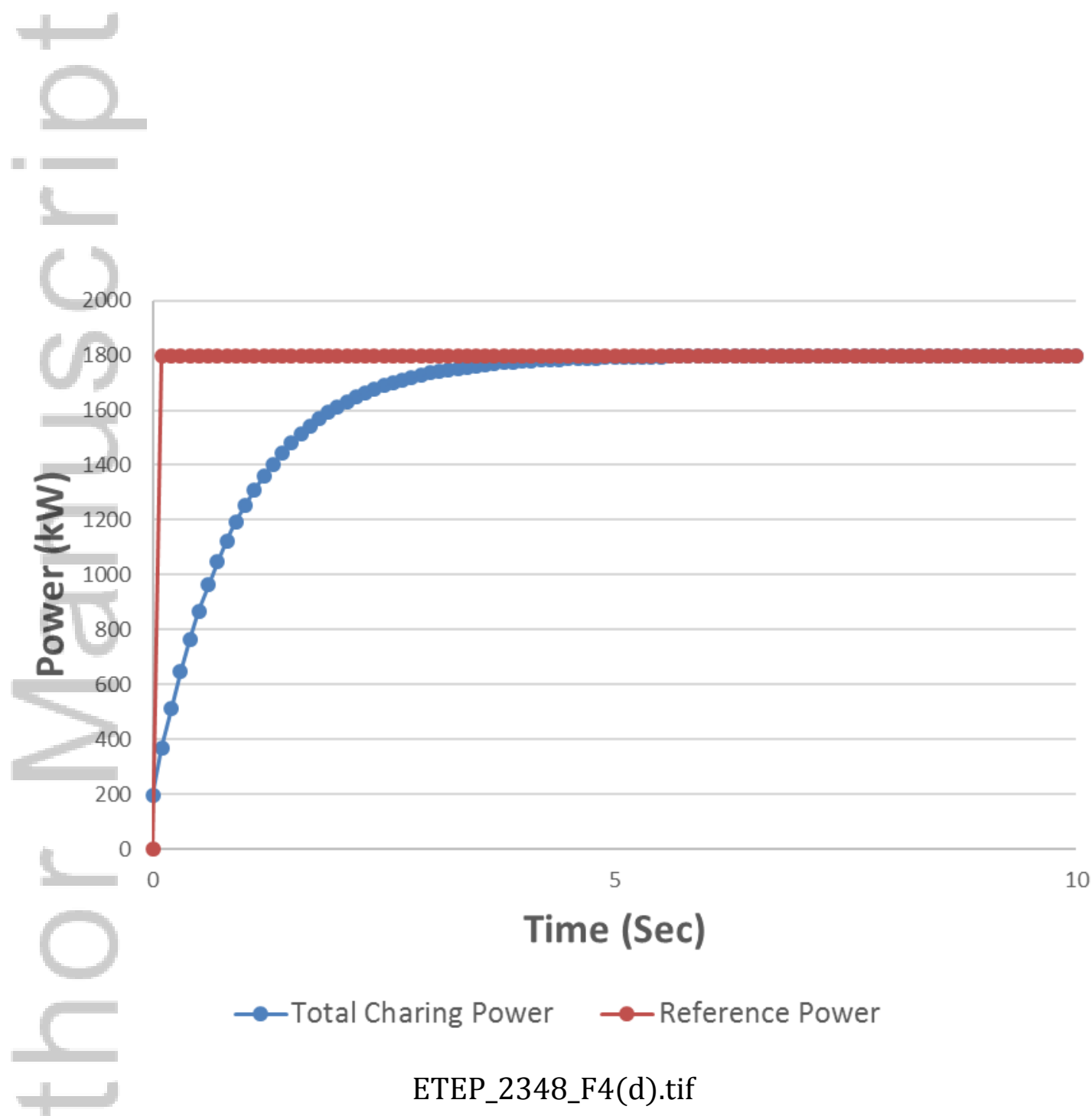

This article is protected by copyright. All rights reserved. 


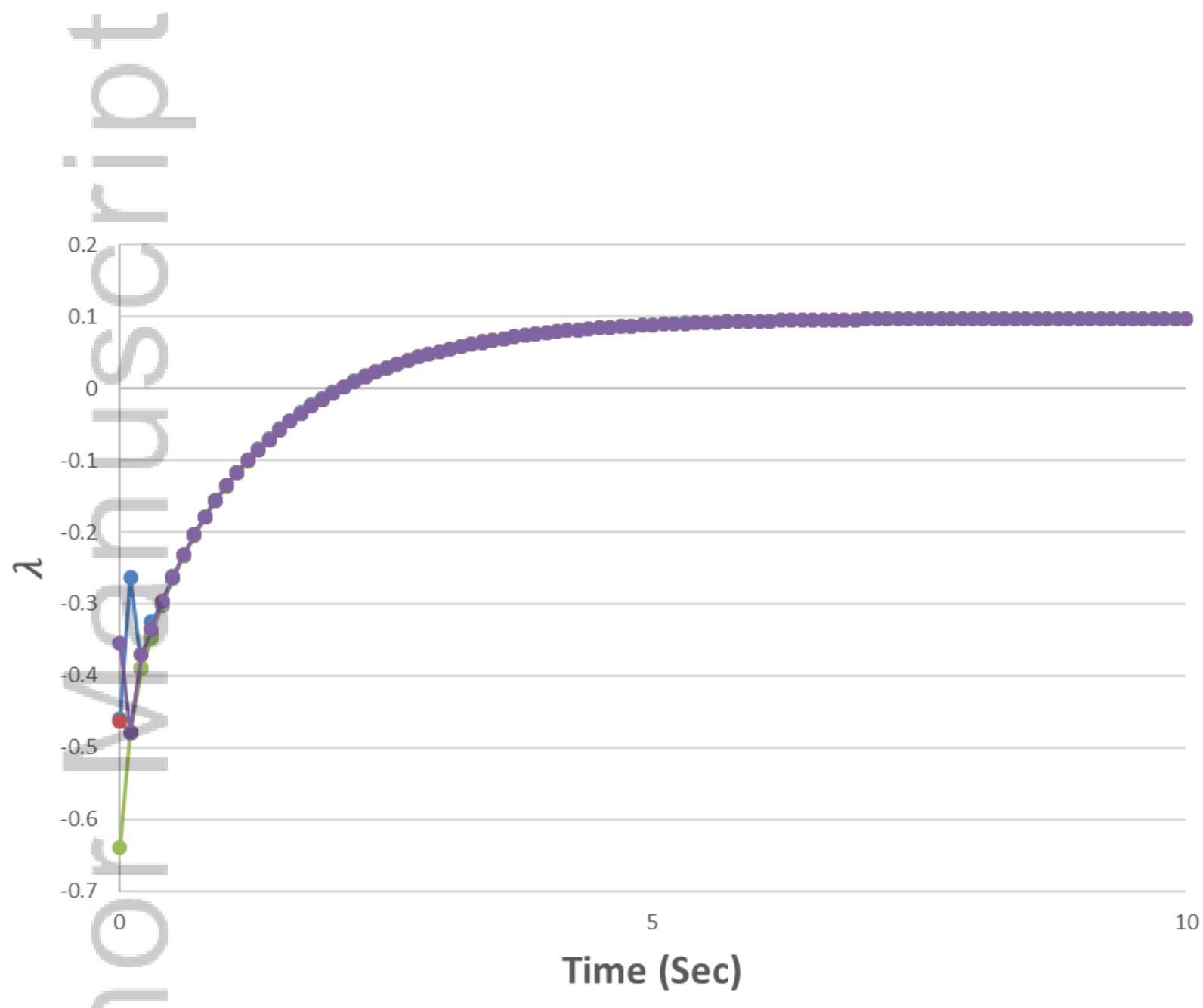

$\longrightarrow$ Parking Deck $1 \multimap$ Parking Deck $2 \multimap$ Parking Deck $3 \multimap$ Parking Deck 4 ETEP_2348_F5(a).tif

This article is protected by copyright. All rights reserved. 

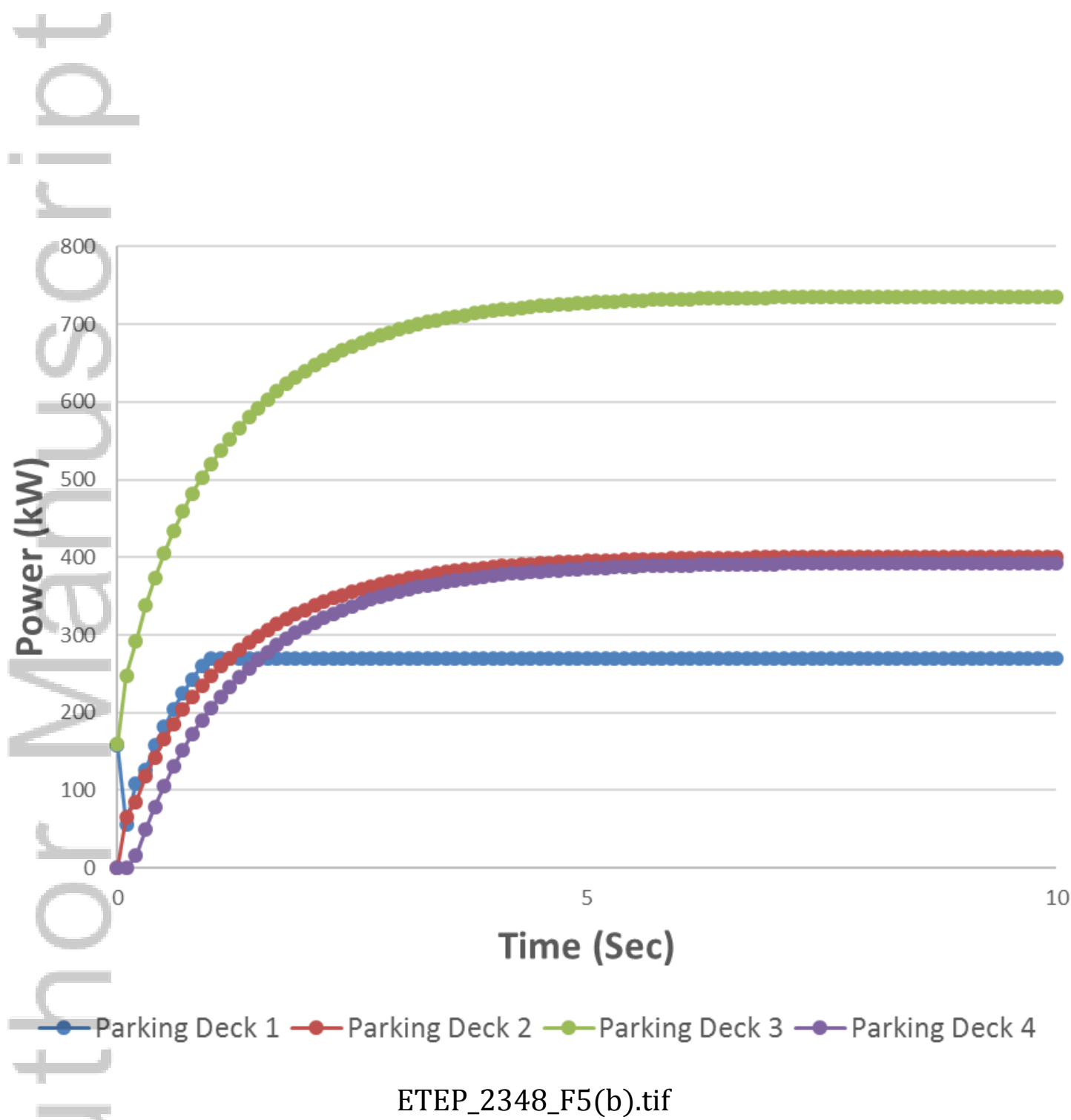

This article is protected by copyright. All rights reserved. 


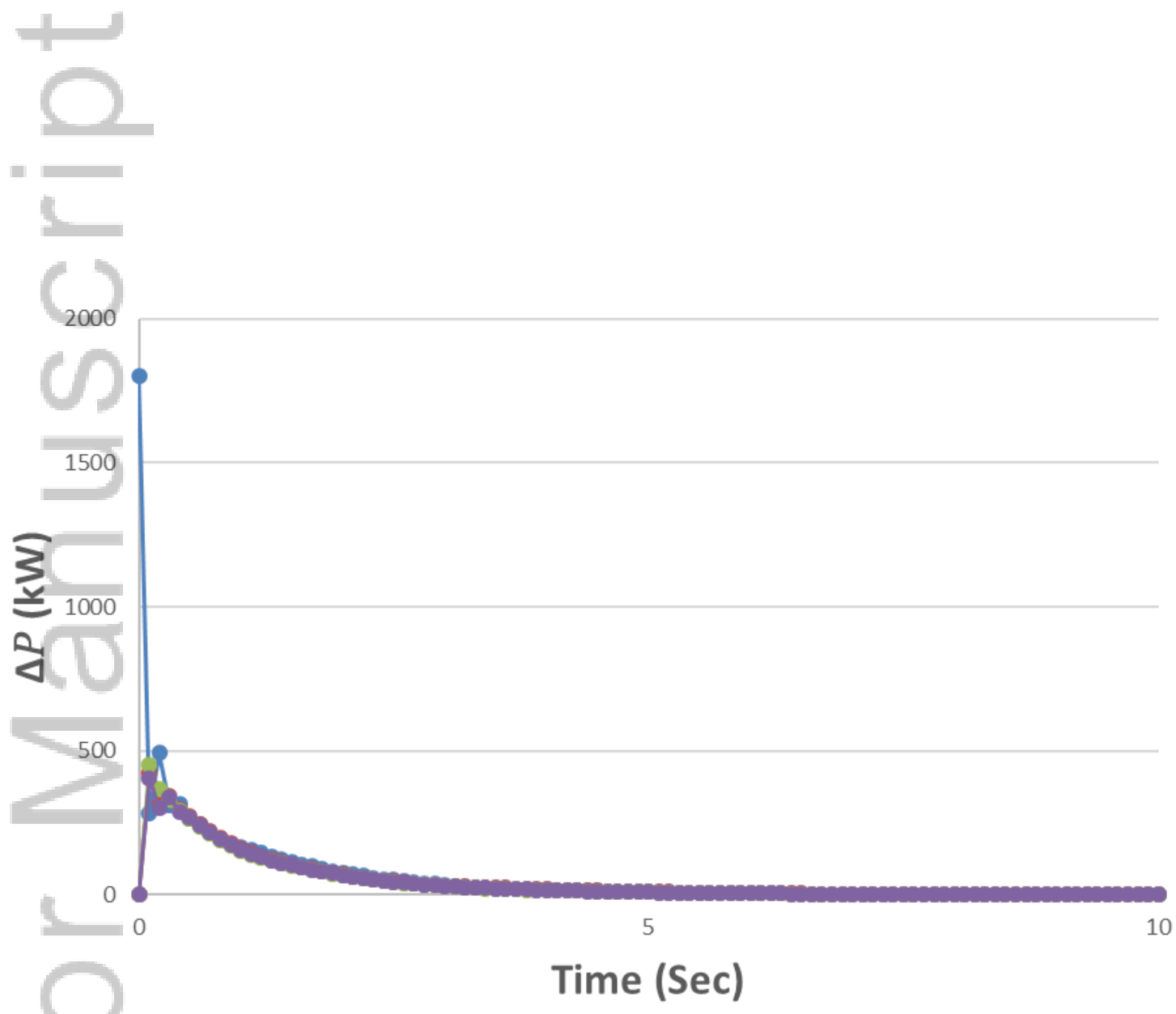

$\longrightarrow$ Parking Deck $1 \rightarrow$ Parking Deck $2 \multimap$ Parking Deck $3 \multimap$ Parking Deck 4

ETEP_2348_F5(c).tif

This article is protected by copyright. All rights reserved. 


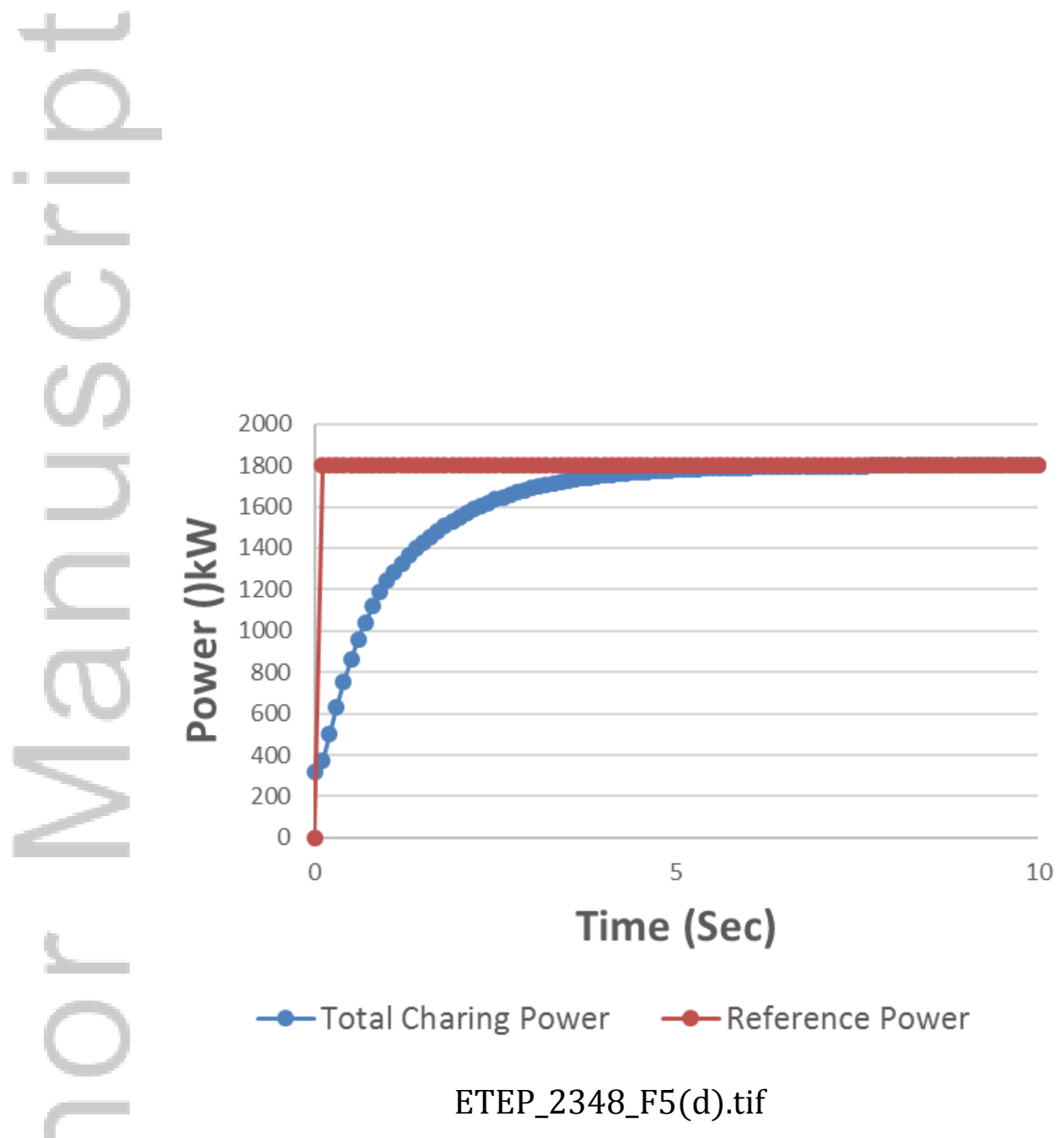

This article is protected by copyright. All rights reserved. 


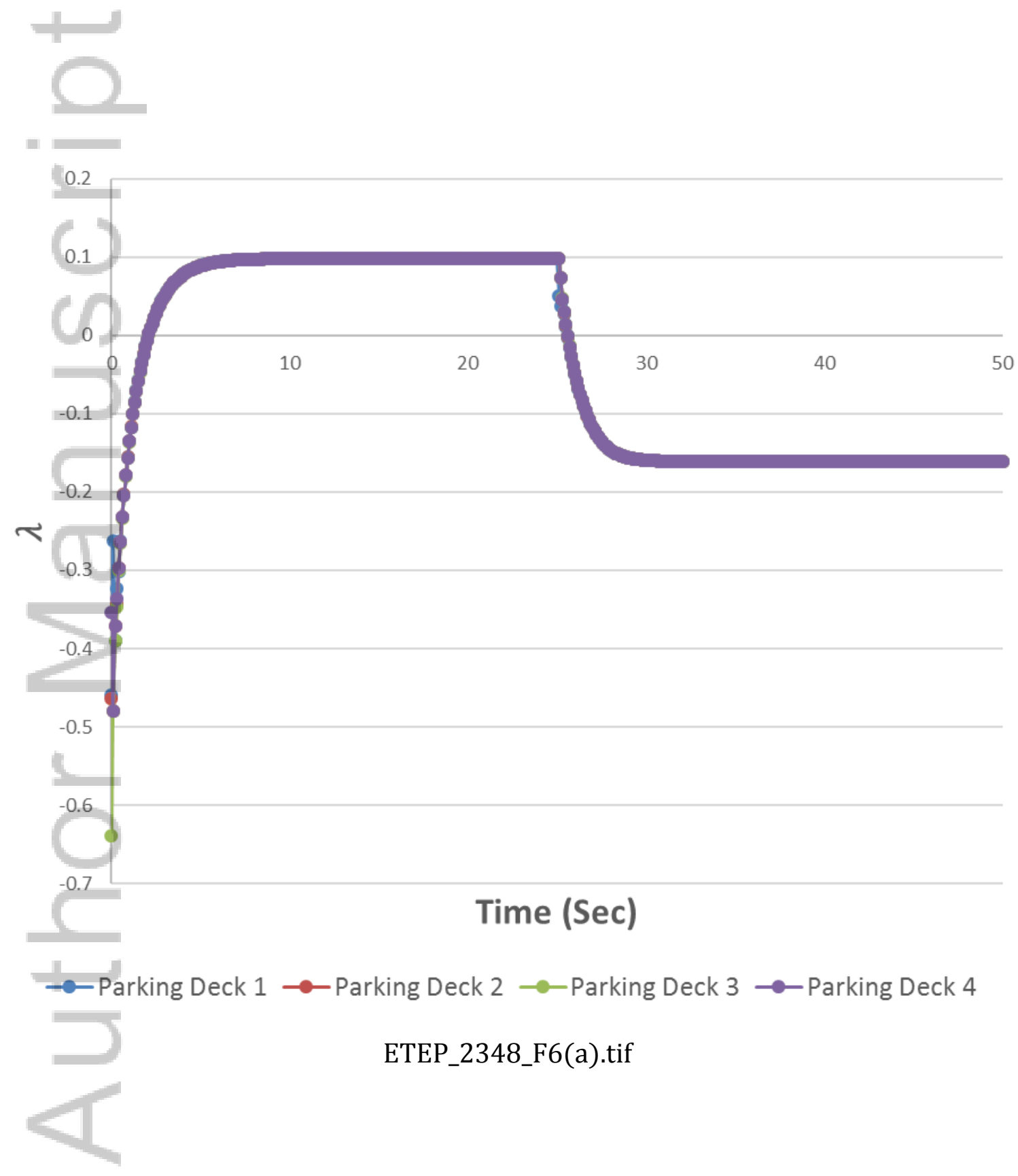

This article is protected by copyright. All rights reserved. 


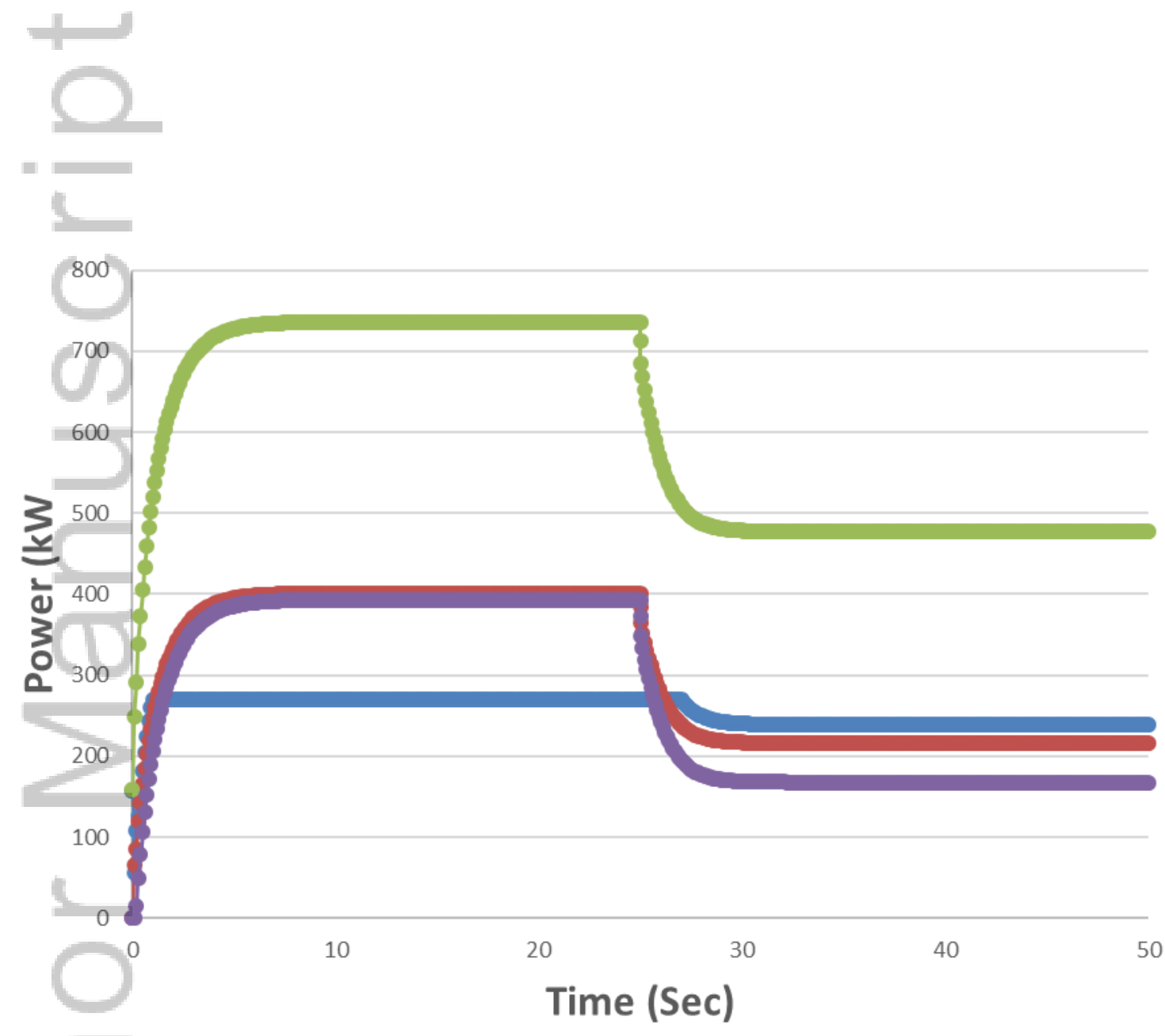

$\multimap$ Parking Deck $1 \multimap$ Parking Deck $2 \multimap$ Parking Deck $3 \multimap$ Parking Deck 4

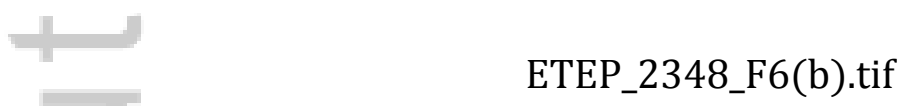

This article is protected by copyright. All rights reserved. 

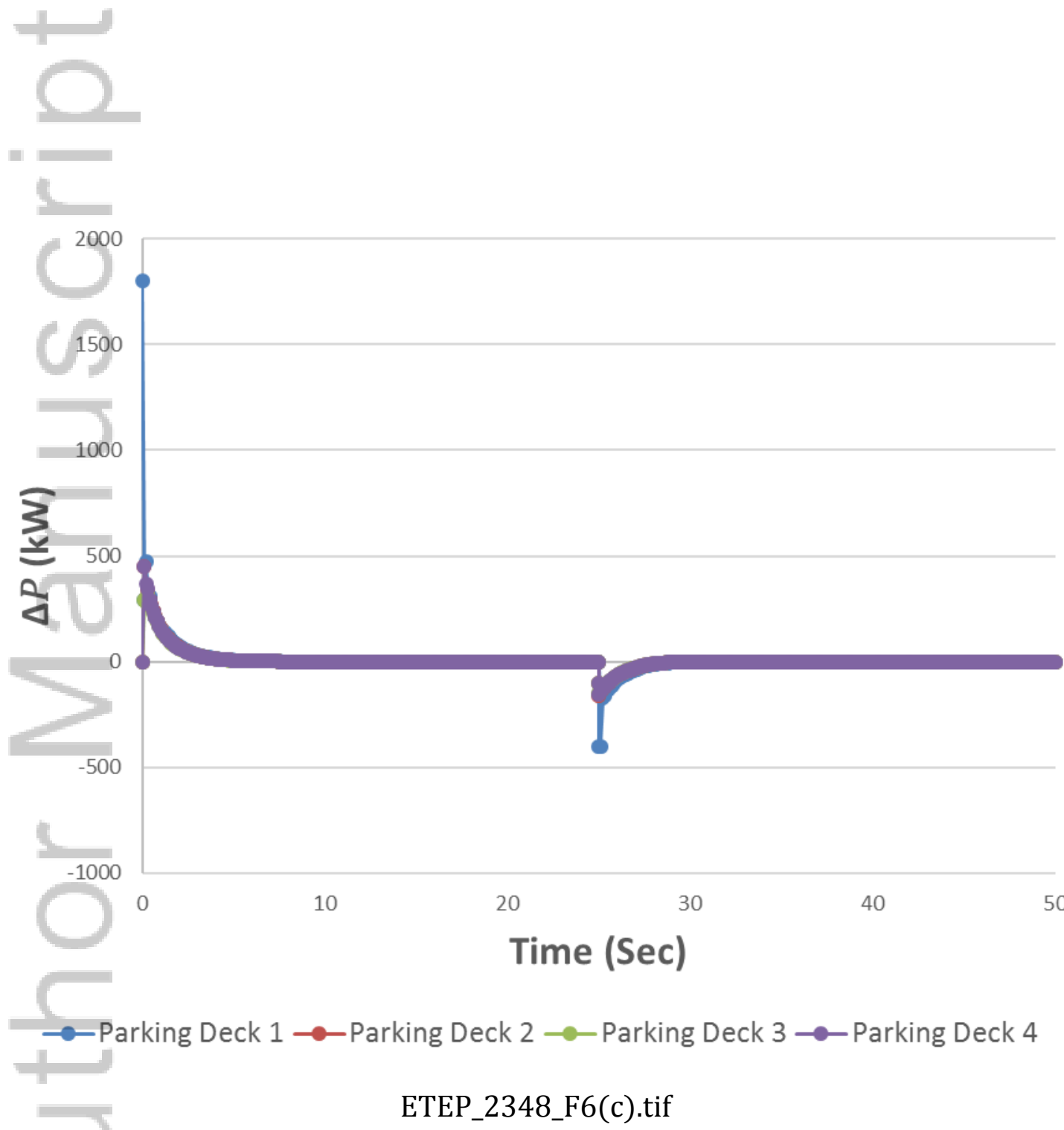

This article is protected by copyright. All rights reserved. 


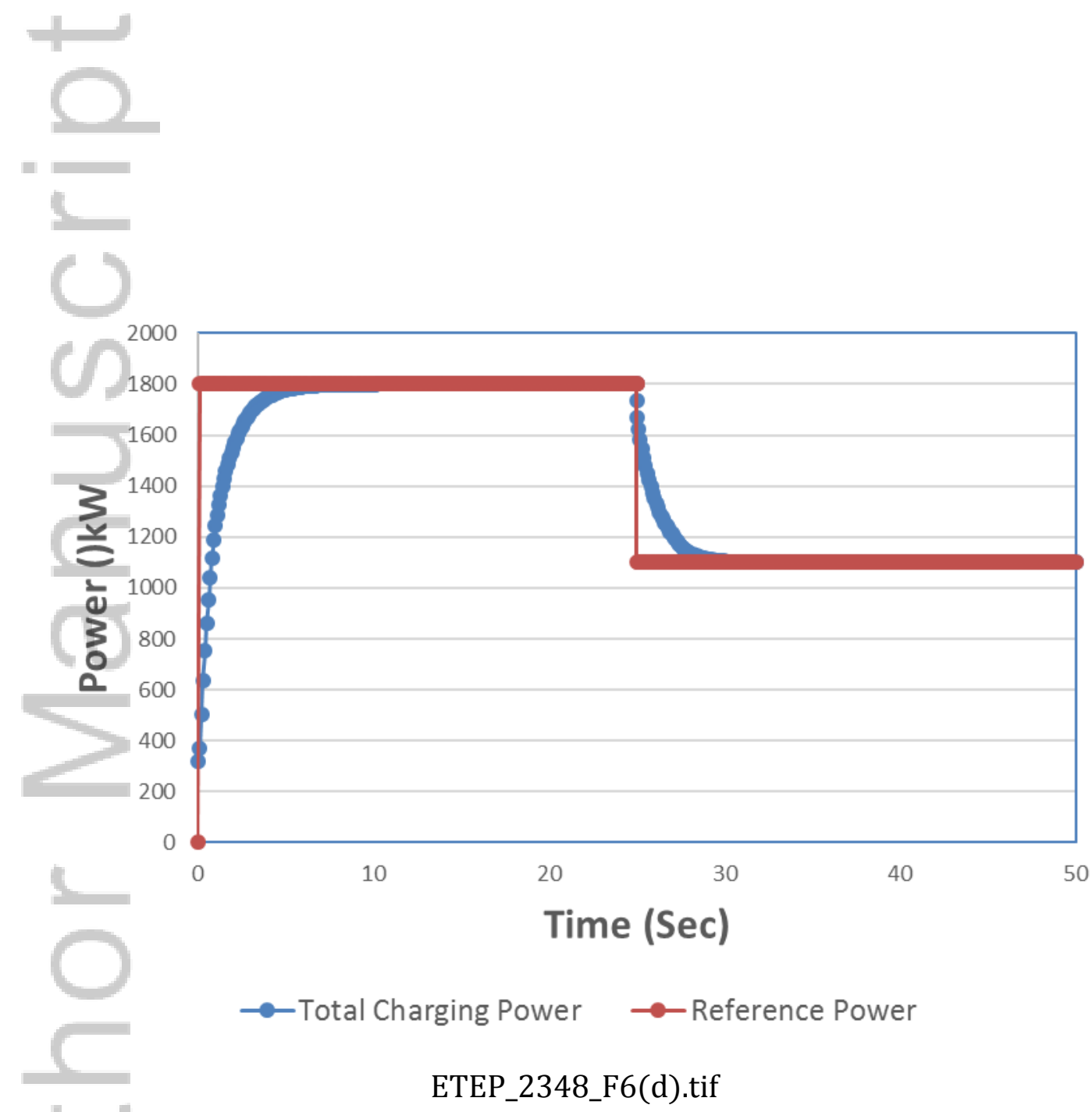

This article is protected by copyright. All rights reserved. 

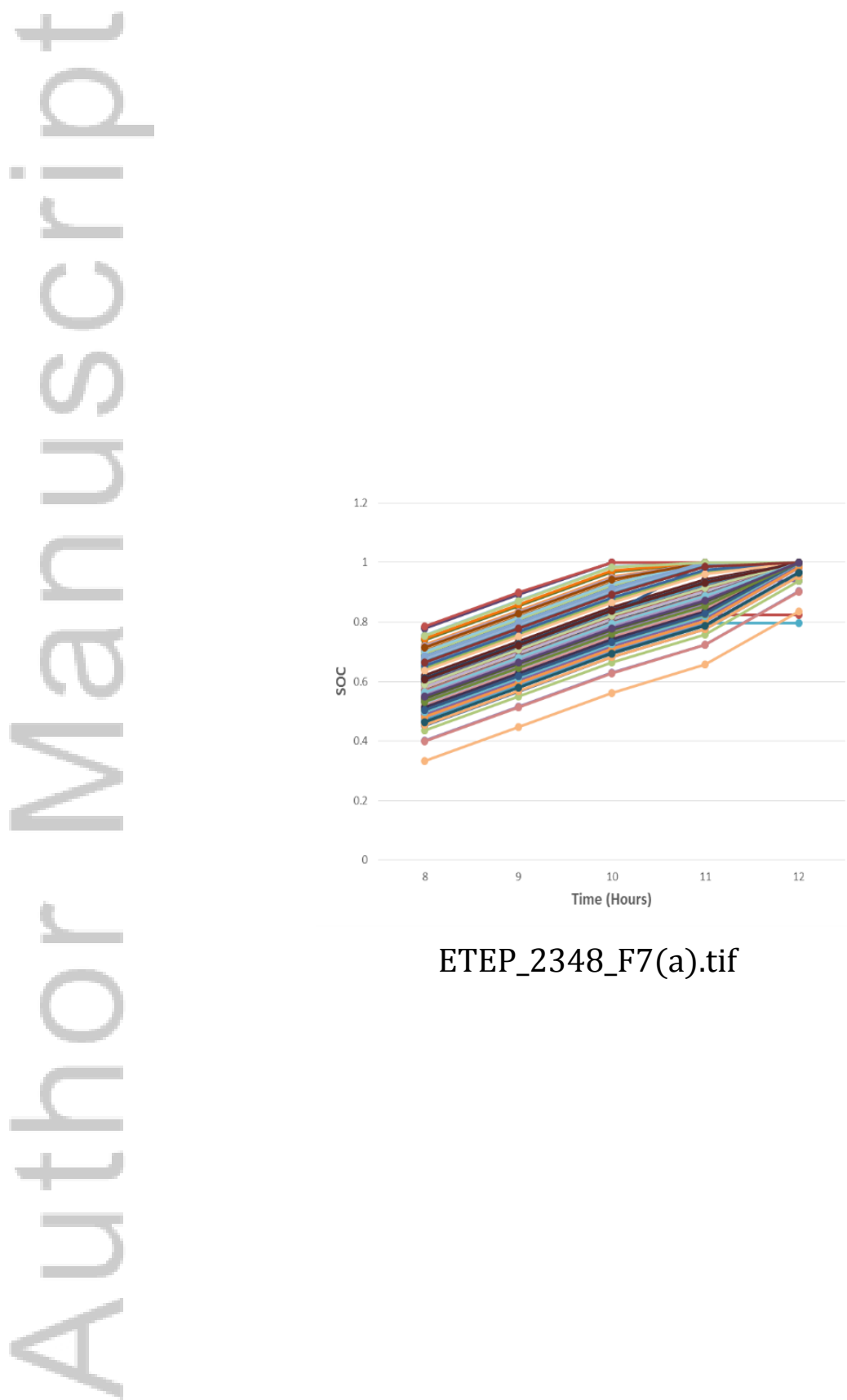

ETEP_2348_F7(a).tif

This article is protected by copyright. All rights reserved. 


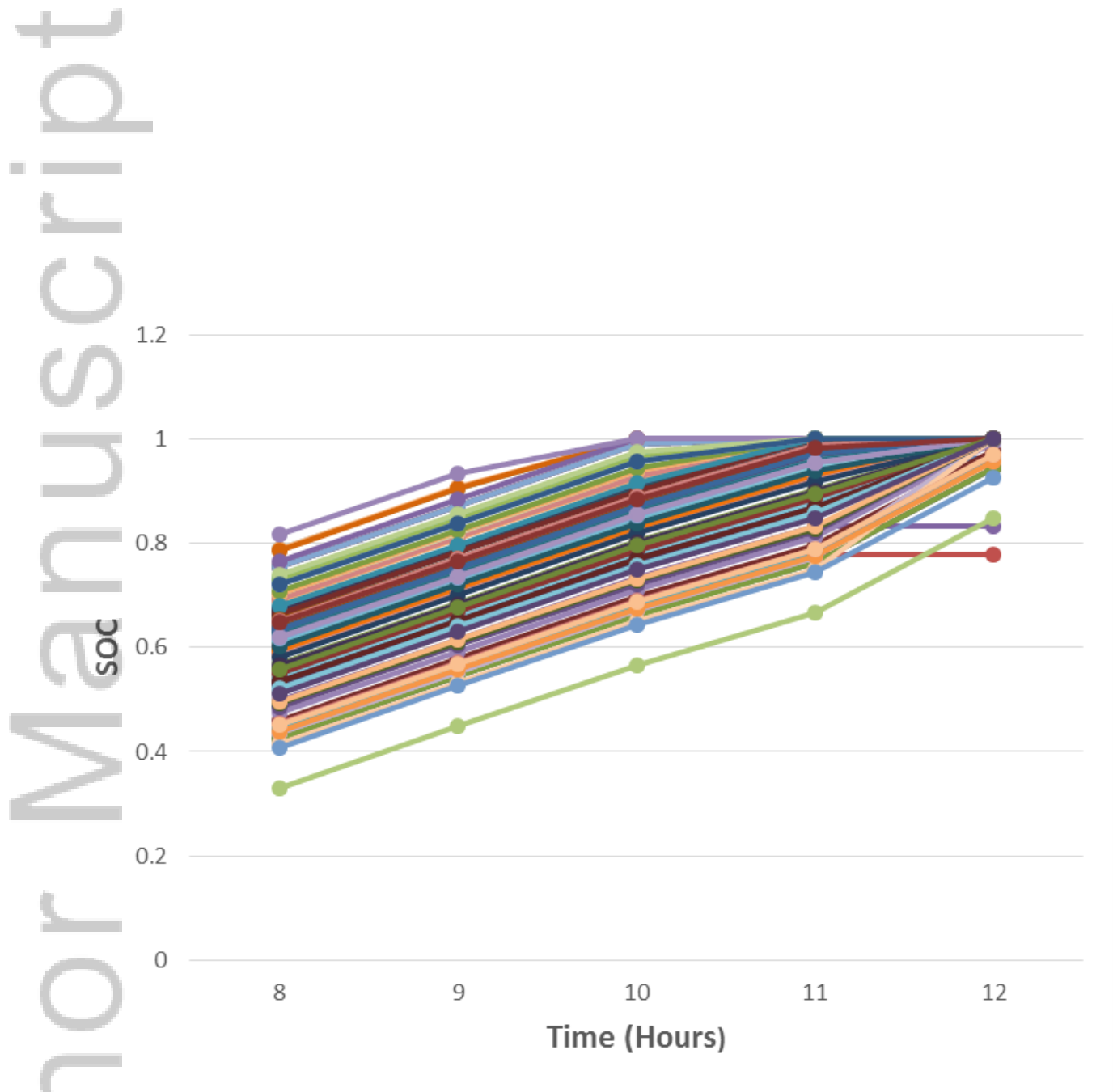

ETEP_2348_F7(b).tif

This article is protected by copyright. All rights reserved. 


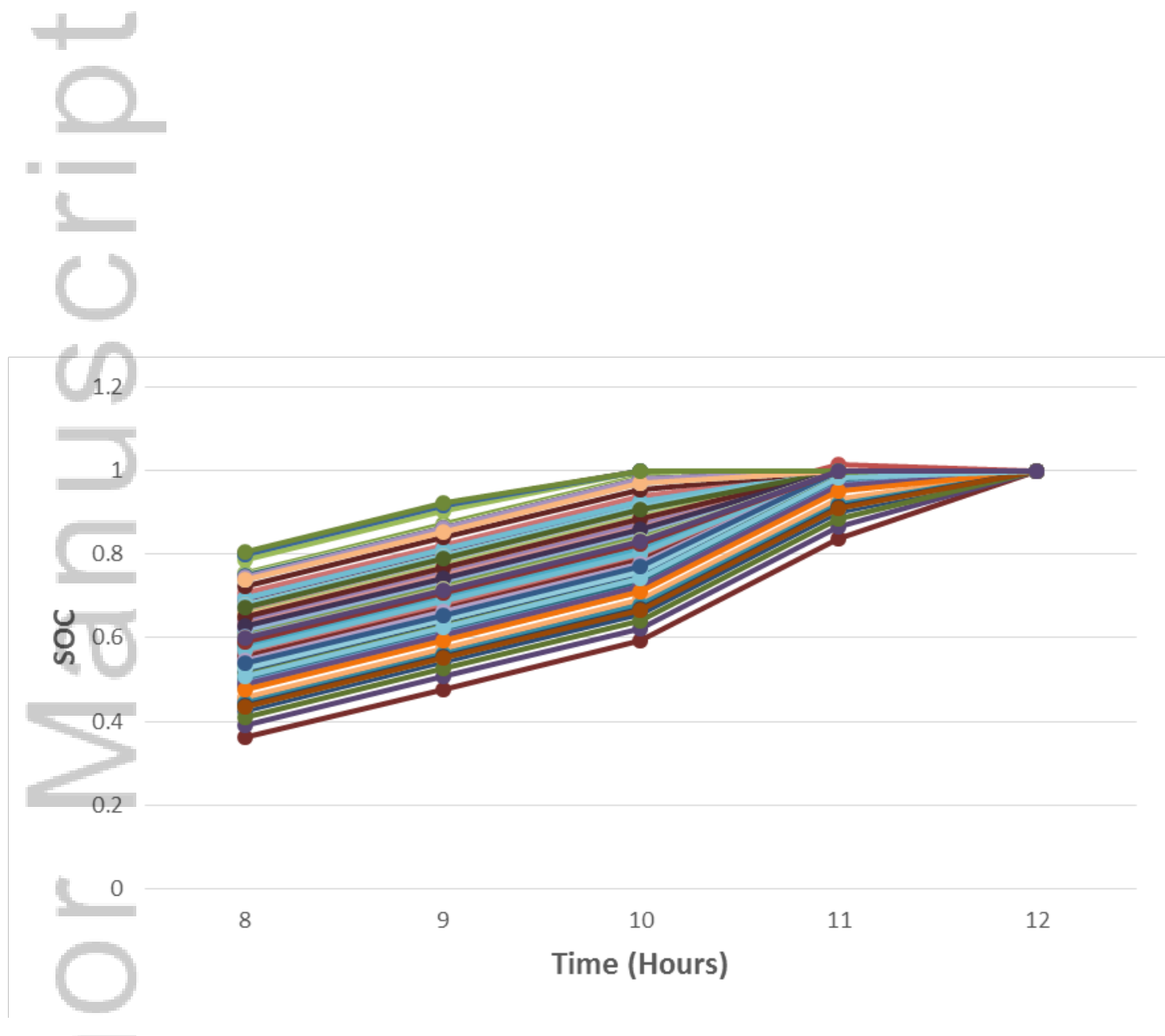

ETEP_2348_F7(c).tif

This article is protected by copyright. All rights reserved. 


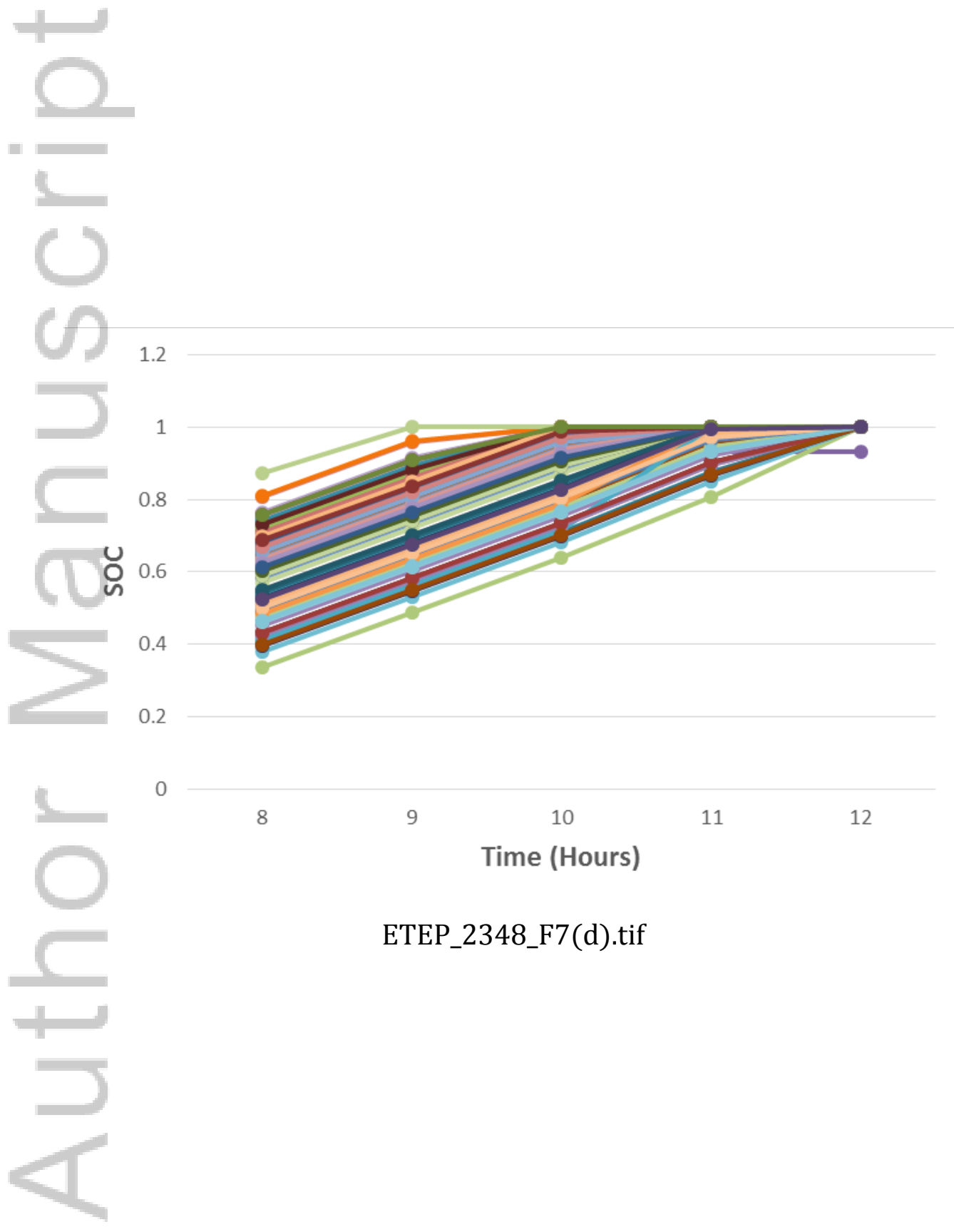

This article is protected by copyright. All rights reserved. 


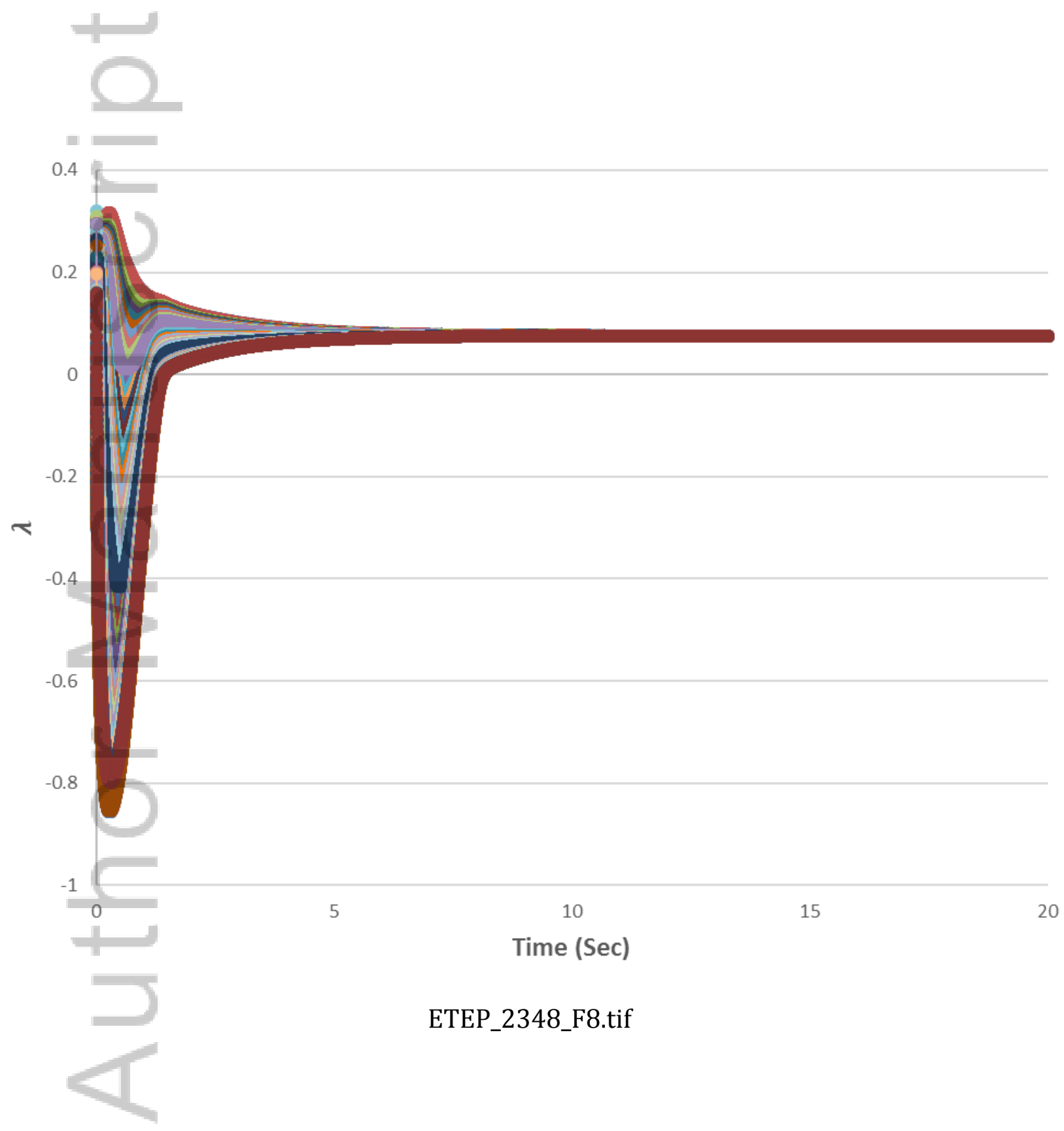

This article is protected by copyright. All rights reserved. 